\title{
Quantization in Polar Coordinates and the Phase Operator
}

\author{
By \\ Daniel A. Dubin*, Mark A. Hennings** and Thomas B. Smith***
}

\begin{abstract}
We review some of the difficulties previously encountered in defining the phase operator for finite quantum systems. We then propose the Wigner-Weyl quantization of the angle function $\varphi$ on phase space as the phase operator, and derive a closed expression for its matrix elements with respect to the Hermite functions. We also determine the quantization of $e^{i \varphi}$, which turns out to be a weighted shift operator, its spectrum and that of its adjoint.

This is done in the framework of quantization of a certain symbol class of phase space distributions, specialized to those which depend on one variable only. After recalling some results for the position and momentum variables, we apply the scheme to functions of radius or angle. We give necessary and sufficient conditions for operators obtained by quantizing functions of the angle to be elements of $\mathscr{L}^{+}[\mathscr{S}(\mathbf{R})]$ and $\mathscr{L}^{+}\left[\mathscr{Y}(\mathbf{R}), L^{2}(\mathbf{R})\right]$, and a sufficient condition for boundedness.

We then consider the associated questions of commutation relations and uncertainties for operators in $\mathscr{L}^{+}\left[\mathscr{S}(\mathbf{R}), L^{2}(\mathbf{R})\right]$, which we define as bilinear forms. As must be the case, the commutator between our phase operator and the number operator exhibits noncanonical terms. Not surprisingly, the Poisson bracket of their phase space symbols also exhibits a noncanonical term.
\end{abstract}

\section{§1. Introduction}

In one of the first papers on quantum electrodynamics, Dirac [1], in considering the Fourier modes of the field, introduced a polar decomposition of the raising and lowering operators (equation (10) and $\S 10$ of [1]). He interpreted the positive self adjoint operator so obtained as the number operator for the mode, and the partial isometry as the exponential of the phase operator. However, a partial isometry which is not unitary cannot be the exponential of an operator, so his interpretation was not quite correct. If one ignores this and proceeds formally, one comes to an operator canonical to the number

Communicated by H. Araki, February 24, 1993.

1991 Mathematics Subject Classifications: 81 S30 (primary); 46N50 (secondary)

* Department of Pure Mathematics, The Open University, Milton Keynes, MK7 6AA, UK

** Sidney Sussex College, Cambridge, CB2 3HU, UK

*** Department of Physics, The Open University, Milton Keynes, MK7 6AA, UK 
operator. This, too, is not possible in the Schrödinger representation, as shown by Susskind and Glogower [2].

Since that time, numerous papers have been written trying to overcome this difficulty. Other than intrinsic mathematical interest, the impetus to do this is that such an operator would provide a convenient mechanism for analyzing certain quantum electrodynamical systems which exhibit classical features in some degree or another, notably quantum optics. The continuing succession of papers concerned with the phase operator testifies to the fact that there is no generally accepted definition for a phase operator. A critique for work up to 1968 may be found in the paper of Carruthers and Nieto [3].

By a canonical phase operator we mean a symmetric map $\Phi \in \mathscr{L}[\mathscr{S}(\mathbf{R})$, $\left.\mathscr{S}(\mathbf{R})^{\prime}\right]$ such that

$$
[\overline{\Phi f}](N g)-[\Phi g](\overline{N f})=i g(\bar{f}), \quad f, g \in \mathscr{S}(\mathbf{R}),
$$

where $N \in \mathscr{L}^{+}[\mathscr{S}(\mathbf{R})]$ is the number operator.

Here and below, we employ the standard notation that if $E$ and $F$ are locally convex spaces, then $\mathscr{L}(E, F)$ is the space of continuous linear maps from $E$ to $F$. The symbol $A \in \mathscr{L}^{+}(E, F)$ indicates that $E$ and $F$ are subspaces of a Hilbert space, and that both $A$ and its Hilbert adjoint $A^{*} \in \mathscr{L}(E, F)$. In this case, $A^{+}$is $A^{*}$ restricted to $E$. If $E=F$ we write $\mathscr{L}(E)$ for $\mathscr{L}(E, E)$ and $\mathscr{L}^{+}(E)$ for $\mathscr{L}^{+}(E, E)$. By $T(f)$ we mean the bilinear duality pairing between $T \in \mathscr{S}(\mathbf{R})^{\prime}$ and $f \in \mathscr{S}(\mathbf{R})$. The connection to our convention for the inner product is that if $f \in \mathscr{S}(\mathbf{R})$ and $g \in L^{2}(\mathbf{R})$, then $g(\bar{f})=\langle f, g\rangle$. The bar indicates the complex conjugate function or distribution. Unless a special point is being made, we shall choose units so that $\hbar=1$.

As noted, no such map $\Phi$ exists [2]. By the existence problem for a phase operator we mean the existence of a map $\Phi \in \mathscr{L}\left[\mathscr{S}(\mathbf{R}), \mathscr{S}(\mathbf{R})^{\prime}\right]$, constructed on the basis of some physical or mathematical principle, whose commutator with $N$ differs from (1.1.a) by terms of order $\hbar$ or higher. If the range of $\Phi$ is $L^{2}(\mathbf{R})$, then we may call it an operator.

We wish to emphasize that the problem we are considering requires the Schrödinger representation. Then the operator domains contain $\mathscr{S}(\mathbf{R})$, which is defined by $N$, and is the natural arena for the unbounded operators of quantum mechanics $[4,5]$. A number of authors have considered the operator of multiplication by the angle on the Hilbert space $L^{2}(T)$ over the circle $[6,7]$, or certain subspaces of it [8]. The resulting operator, which we shall denote $\Delta(\Xi)$, can then be transported to the Schrödinger representation, which we shall do in the third section. In this way $\Delta(\Xi)$ is seen to be an operator which is not the quantization of a function of the angle alone, as discussed below. For this reason we are not convinced of its physical provenance. We take the position that phase operators obtained through representations other 
than the Schrödinger representation do not solve the existence problem.

Some authors, notably Barnett and Pegg [9] and Popov and Yarunin [7], have considered replacing the phase operator by a sequence of rank $n$ operators, taking the limits of certain matrix elements. (They have, in fact. considered a sequence of operators on an ascending sequence of finite dimensional Hilbert spaces, but that comes to the same thing.) The authors in [9] leave open the question of the convergence of this sequence of operators in some suitable sense, but this point has been considered by Popov and Yarunin [7]. Their result is the operator $\Delta(\Xi)$ mentioned above.

A related problem is to construct a bounded operator $U \in \mathscr{L}^{+}[\mathscr{S}(\mathbf{R})]$ satisfying the integrated form of the commutation relation for number and phase:

$$
[U, N]_{-} f=U f, \quad f \in \mathscr{S}(\mathbf{R}) .
$$

In contrast with the phase operator problem, this has many solutions, and the difficulty is in choosing one with particular significance. Of course there is no solution which is unitary, for if there were, its generator would be a canonical phase operator [2].

The earliest solution of (1.1.b) was obtained from the polar decomposition of the lowering operator [1]. An interesting class of them has been introduced by Lerner, Huang and Walters [10], namely weighted shift operators

$$
U_{\lambda} h_{n}=\lambda_{n} h_{n-1} \text { and } U_{\lambda}^{*} h_{n}=\lambda_{n+1} h_{n+1},
$$

where the $h_{n}, n \geq 0$, are the orthonormal Hermite basis for $L^{2}(\mathbf{R})$, and the sequence $\lambda=\left(\lambda_{n}\right)_{n \geq 0}$ satisfies $\lambda_{0}=0$; is bounded; $\lambda_{n}>0$ for all $n \geq 1$; the $\left(\lambda_{n}\right)_{n \geq 0}$ increase monotonically to unity: $\lim _{n \rightarrow x} \lambda_{n}=1$; and satisfy a chain condition:

$$
\frac{1}{4} \lambda_{n}^{2}=g_{n}\left(1-g_{n-1}\right), \quad 0<g_{n}<1
$$

We note that the first two conditions are sufficient to guarantee that the operators $U_{\lambda}$ and $U_{\lambda}^{*} \in \mathscr{L}^{+}[\mathscr{S}(\mathbf{R})]$ and satisfy the commutation relations with the number operator. The other conditions [10] are used to find the spectra of the "cosine" and "sine" operators

$$
C_{i}=\frac{1}{2}\left(U_{i}+U_{i}^{*}\right) \quad \text { and } \quad S_{i}=\frac{1}{2 i}\left(U_{i}-U_{i}^{*}\right)
$$

The justification advanced in [10] for considering this class of sequences is based on the resulting spectra of $C_{i}$ and $S_{\lambda}$, and that the $\lambda$ are very much like the sequence $\lambda_{n}=1$ for $n \geq 1$ which comes from the lowering operator. But these considerations do not succeed in selecting one solution from amongst the 
others, nor do any of the sequences $\lambda$ have any particular merit over any other. In contrast, our quantization procedure applied to the phase space function $e^{ \pm i \varphi}$ results in a particular weighted shift operator $\Delta\left(e^{ \pm i \varphi}\right)$ of the form (1.2), though not one satisfying the conditions proposed in [10], cf, (3.17.b) below.

Other authors have constructed the operator $\Delta(\Xi)$ in interesting and unusual settings. Amongst these we note the work of Newton [11] and Ban [12]. Newton doubles up the system Hilbert space, considering $\mathscr{H}=L^{2}(\mathbf{R}) \oplus$ $L^{2}(\boldsymbol{R})$, and thereby constructs a unitary extension $V$ of the partial isometry $U$. Utilizing the isomorphism between $\mathscr{H}$ and $l^{2}(\mathbf{Z})$ afforded by two copies of the Hermite basis elements, this unitary extension $V$ may be identified with the bilateral shift operator. The spectral theorem for $V$ represents it as the multiplication operator $e^{-i \theta}$ on $L^{2}([-\pi, \pi))$. Hence its generator is well defined and self adjoint, and is the multiplication operator $\theta$ in the spectral representation.

One must now project back to the original Hilbert space. The projection of the generator of $V$ is then the proposed phase operator. A calculation shows that this is the now familiar operator $\Delta(\Xi)$. We see, then, that while this approach is interesting and useful for certain purposes, it sheds no light on the existence problem for a phase operator beyond the considerations previously discussed.

Ban considers a reservoir-system set-up as in statistical mechanics. This is evidently an interesting and useful formalism for quantum optics, but it, too, does not go beyond $\Delta(\Xi)$ for the system alone when projected back. We might point out that, up to an isomorphism, Ban's universe is a countable number of copies of the Hilbert space $\mathscr{H}$ used by Newton.

These considerations apply to a finite number of degrees of freedom only. For an infinite number of degrees of freedom the situation is quite different. For Weyl systems there may or may not be a global number operator. If there is, the representation admits a factorization into a tensor product of a finite number of copies of the Schrödinger representation and a trivial infinite dimensional representation, and in such cases the situation is the same as for $f^{2}$ degrees of freedom [13]. But there are models of relevance to statistical mechanics where a number density operator exists and phase effects are important. These are associated with superconductivity and superfluidity, and are not relevant to the problem at hand [13-16].

Since a phase operator cannot be canonical, and cannot be defined by a weighted shift operator, on what basis should it be defined? We propose that basis to be quantization in the sense of Wigner and Weyl, or harmonic analysis on phase space. [17-29].

In Section 2 we introduce the relevant quantization scheme, which associates with any distribution $T \in \mathscr{S}(\Pi)^{\prime}$ on phase space $\Pi$ a map 
$\Delta(T) \in \mathscr{L}\left[\mathscr{S}(\mathbf{R}), \mathscr{S}(\mathbf{R})^{\prime}\right]$. We then consider quantization of phase space functions of suitable symbol class [29], then specializing to an associated symbol class of one variable. We find conditions for the resulting operators to be members of $\mathscr{L}^{+}[\mathscr{S}(\mathbf{R})]$ and $\mathscr{L}^{+}\left[\mathscr{S}(\mathbf{R}), L^{2}(\mathbf{R})\right]$.

When our quantization scheme is applied to functions of position and momentum alone, the results are completely standard, and we note these in passing. Applied to functions of the radius the results are also known, cf, $\S 1.9$ of Folland's text [29]. We provide some details of this notwithstanding, since the quantization of functions of the polar angle is set up in the same way.

In Section 3 we consider quantization of functions of the angle. The results in this case are more complicated than for functions of $p, q$ or $r$. We derive an expression for the matrix elements of $\Delta(T)$, give a necessary and sufficient condition for it to belong to $\mathscr{L}^{+}[\mathscr{S}(\mathbf{R})]$, a sufficient but not necessary condition for it to be bounded, and a necessary and sufficient condition for it to belong to $\mathscr{L}^{+}\left[\mathscr{S}(\mathbf{R}), L^{2}(\mathbf{R})\right]$. We show that $\Delta\left(e^{ \pm i \varphi}\right) \in \mathscr{L}^{+}[\mathscr{S}(\mathbf{R})]$ and is bounded, and that $\Delta(\varphi) \in \mathscr{L}^{+}\left[\mathscr{S}(\mathbf{R}), L^{2}(\mathbf{R})\right]$ but not $\mathscr{L}^{+}[\mathscr{S}(\mathbf{R})]$.

These results are found by considering the matrix representation of these operators with respect to the Hermite basis. Subsequently, in Section 5, we use the kernel representation of the angle operators to find a sharper sufficient condition for boundedness. In the particular case of $\Delta(\varphi)$ we can do better, and prove that it is bounded, with norm $\|\Delta(\varphi)\| \leq 3 \pi / 2$.

In Section 3 we present an analysis of the spectra of the operators $\Delta\left(e^{-i \varphi}\right)$ and $\Delta\left(e^{i \varphi}\right)$, following [10]. We do not, however, find the spectrum of the phase operator, although we conjecture that it is purely continuous, covering $[-\pi, \pi]$. Thus the work in Section 3 implements our main idea, which is that the quantization $\Delta(\varphi)$ of $\varphi$ is a good candidate for a phase operator.

We have not found any similar work in the literature, and so our results may be of some interest in the theory of harmonic analysis in phase space [29] as well as in physics.

We wish to point our that the angle function is not periodic, and its definition requires a certain care, since the pole in polar coordinates is actually an essential singularity requiring a branch cut. This branch point singularity is reflected in several ways. For one thing, the Poisson bracket between the angle and the function $\frac{1}{2}\left(r^{2}-1\right)$, whose quantization is the number operator. must be treated as a distribution. The result differs from 1 by a distribution concentrated at $q=0$. We refer to this non-canonical term as secular. This is an important new observation, as it is generally held that these functions are canonically conjugate [13].

The branch point is also reflected in the procedure necessary to determine its kernel. As this procedure involves a detailed application of Lebesgue integration theory, we have relegated it to an appendix. 
In Section 4 we use the results of the appendix to present the kernels of the operators corresponding to $\varphi, e^{ \pm i \varphi}$ and $r^{2}$. We then consider the commutator of $\Delta(\varphi)$ and the number operator. Because of domain problems, the commutator has been defined as a sesquilinear form $[30,31]$. The resulting form differs from canonicity by terms of higher order in $\hbar$ which we have called secular in analogy with the classical result. Thus the naive consideration that number and phase form a canonical pair classically and quantum mechanically must be modified in both theories.

As noted above, Section 5 contains an analysis of a sufficient condition for boundedness of angle operators.

This paper follows on from a previous one [32] in which it was proposed that the quantization of the angle function in phase space be a candidate for a phase operator. In that work we derived the matrix elements of the phase operator, but in a less concise form than here and proceeded more symbolically, emphasizing the physical consequences. The material here serves to justify and extend the results noted there.

\section{§2. Phase Space Quantization}

Although phase space quantization is well known [27-29], in this section we shall write down a few of the basic formulas with a view towards establishing our notation.

By phase space quantization we shall mean associating to each tempered distribution $T$ on phase space a mapping from $\mathscr{S}(\mathbf{R})$ to $\mathscr{S}(\mathbf{R})^{\prime}$, denoted $\Delta(T)$, following a well known generalization of the prescription of Weyl [27].

To be precise, let us write $\Pi$ for $\mathbf{R}^{2}$ interpreted as phase space, and let $T \in \mathscr{S}(\Pi)^{\prime}$ be a tempered distribution. Then for $\Delta(T)$ we take

$$
[\Delta(T) g](f)=T[\mathscr{G}(f \otimes g)], \quad f, g \in \mathscr{S}(\mathbf{R}), T \in \mathscr{S}(\Pi)^{\prime},
$$

where $\mathscr{G}: \mathscr{S}\left(\mathbf{R}^{2}\right) \rightarrow \mathscr{S}(\Pi)$ is the transformation

$$
\mathscr{G}[F](p, q)=(2 \pi)^{-1} \int_{\mathbf{R}} F\left(q+\frac{1}{2} x, q-\frac{1}{2} x\right) e^{i p x} d x .
$$

We note that $\mathscr{G}$ is an isomorphism and $\Delta(T)$ is linear and continuous.

To understand the notation the reader must note that $f$ and $g$ are smooth wave functions in the system Hilbert space $L^{2}(\mathbf{R})$ and $T$ is the phase space distribution which is being quantized. It should be noted that $\mathscr{G}$ extends to a continuous bijection from $L^{2}\left(\mathbf{R}^{2}\right)$ to $L^{2}(\Pi)$.

A characteristic feature of this theory is that a direct operator form can be given for the mapping between $\mathscr{S}(\Pi)^{\prime}$ and $\mathscr{L}\left[\mathscr{S}(\mathbf{R}), \mathscr{S}(\mathbf{R})^{\prime}\right]$, at least in certain cases.

Namely, let us define the operator $\Delta(p, q)$ for each $(p, q) \in \Pi$ by 


$$
[\Delta(p, q) f](x)=2 e^{2 i p(x-q)} f(2 q-x), \quad f \in \mathscr{S}(\mathbf{R}), x \in \mathbf{R},(p, q) \in \Pi .
$$

Symbolically, $\Delta(p, q)$ can be written as the Fourier transform of the Weyl operator :

$$
\Delta(p, q)=\frac{1}{2 \pi} \int_{\mathbf{R}^{2}} e^{i(p u+q v)} e^{-i(u P+v Q)} d u d v
$$

so that

$$
\langle f, \Delta(p, q) g\rangle=2 \pi \mathscr{G}(\bar{f} \otimes g)(p, q) .
$$

It is a temptation to write, as do many physicists [23],

$$
\Delta(T)=\frac{1}{2 \pi} \int_{\Pi} T(p, q) \Delta(p, q) d p d q
$$

for suitable $T$. Although very suggestive, this equation requires a proof that the operator-valued integral be well defined in a suitable fashion. In fact, this equation converges weakly in the sense that

$$
[\Delta(T) g](\bar{f})=\frac{1}{2 \pi} \int_{\Pi} T(p, q)\langle f, \Delta(p, q) g\rangle d p d q, \quad f, g \in \mathscr{S}(\mathbf{R}) .
$$

We shall not use $\Delta(p, q)$ directly, but it is worth noting that formal application of these equations often leads to the correct answer in the simplest fashion.

In certain cases it is known what operator class $\Delta(T)$ belongs to for special classes of distributions. The standard results are these:

(i) If $T \in L^{2}(\Pi)$, then $\Delta(T)$ is a Hilbert-Schmidt operator on $L^{2}(\mathbf{R})$, and $T \rightarrow \sqrt{2 \pi} \Delta(T)$ is unitary $[24,25]$ from $L^{2}(\Pi)$ onto the space of Hilbert-Schmidt operators on $L^{2}(\mathbf{R})$ :

$$
\langle T, S\rangle=2 \pi \operatorname{tr}\left(\Delta(T)^{*} \Delta(S)\right) ;
$$

(ii) If $T \in L^{1}(\Pi)$, then $\Delta(T)$ is compact [26];

(iii) If $T \in \mathscr{S}(\Pi)$, then $\Delta(T) \in \mathscr{L}^{+}[\mathscr{S}(\mathbf{R})]^{\prime}$ is a functional on the algebra $\mathscr{L}^{+}[\mathscr{S}(\mathbf{R})]$, such that $T \rightarrow \Delta(T)$ is a linear and topological isomorphism [18]. Recall that $\mathscr{L}^{+}[\mathscr{S}(\mathbf{R})]^{\prime}$ is the space of all linear maps $A \in \mathscr{L}^{+}[\mathscr{S}(\mathbf{R})]$ such that $B A C$ is closeable with trace class closure for all $B, C \in \mathscr{L}^{+}[\mathscr{S}(\mathbf{R})]$. The formula

$$
A, B \longrightarrow \operatorname{tr}(A \bar{B})
$$

defines the duality between $A \in \mathscr{L}^{+}[\mathscr{S}(\mathbf{R})]^{\prime}$ and $B \in \mathscr{L}^{+}[\mathscr{S}(\mathbf{R})]$.

We have not distinguished between functions and the distributions they 
define, but that should occasion no misunderstanding.

Let us introduce our phase space symbol class $[29] \mathbb{C}^{x}(\Pi)$ :

$$
\mathbb{C}^{\infty}(\Pi)=\bigcup_{n=0}^{\infty} \mathcal{C}^{(1)}(\Pi),
$$

where

$$
\mathbb{C}^{(n)}(\Pi)=\left\{F: \Pi \rightarrow \mathbf{C}:(p, q) \rightarrow F(p, q)\left(1+p^{2}\right)^{-n}\left(1+q^{2}\right)^{-n} \in L^{2}(\Pi)\right\} .
$$

Along with this class we shall consider an associated symbol class of one variable functions,

$$
\mathbb{C}^{\infty}(\mathbf{R})=\bigcup_{n=0}^{\infty} \mathcal{C}^{(n)}(\mathbf{R})
$$

where

$$
\mathcal{C}^{(n)}(\mathbf{R})=\left\{\mathfrak{f}: \mathbf{R} \rightarrow \mathbf{C}: x \rightarrow \mathfrak{f}(x)\left(1+x^{2}\right)^{-n} \in L^{2}(\mathbf{R})\right\} .
$$

It is immediate that if $\mathfrak{f} \in \mathcal{C}^{x}(\mathbf{R})$, then $\mathfrak{i} \otimes I$ and $I \otimes \mathfrak{f} \in \mathcal{C}^{(1)}(\Pi)$, where

$$
[\mathfrak{f} \otimes I](p, q)=\mathfrak{f}(p) \quad \text { and } \quad[I \otimes \mathfrak{f}](p, q)=\mathfrak{f}(q), \quad(p, q) \in \Pi .
$$

If $\mathfrak{f} \in \mathcal{C}^{(1)}(\mathbf{R})$, we can define two, related, operators $\mathfrak{f}(Q), \mathfrak{f}(P) \in \mathscr{L}^{+}[\mathscr{S}(\mathbf{R})$, $\left.L^{2}(\mathbf{R})\right]$, in the usual way:

$$
[\mathfrak{f}(Q) g](x)=\mathfrak{f}(x) g(x) \text { and }[\mathscr{F} \mathfrak{f}(P) g](x)=\mathfrak{f}(x)[\mathscr{F} g](x), \quad g \in \mathscr{S}(\mathbf{R}), x \in \mathbf{R} \text {. }
$$

For the special cases where $\mathfrak{f}$ is a polynomial, $\mathfrak{f}(Q)$ and $\mathfrak{f}(P)$ are elements of $\mathscr{L}^{+}[\mathscr{S}(\mathbf{R})]$, since multiplication by $\left(1+x^{2}\right)^{n} /\left(1+x^{2}\right)^{n}$ turns $\mathfrak{f}(x) g(x)$ into a product of an $L^{2}$ and an $L^{x}$ function.

The connection to phase space quantization comes from noting that

$$
T=\left\{\begin{array}{l}
\mathfrak{f} \otimes I \\
I \otimes \mathfrak{f}
\end{array}\right\} \text { implies } \Delta(T)=\left\{\begin{array}{c}
\mathfrak{f}(P) \\
\mathfrak{f}(Q)
\end{array}\right\}, \quad \text { respectively, } \tilde{\mathfrak{f}} \in \mathbb{C}^{x}(\mathbf{R}) .
$$

Going over from Cartesian to polar coordinates in phase space, we consider next functions of the radius alone. As Folland points out [29], many results concerning quantization of radial functions have been independently discovered and rediscovered by various authors [33-38]. Nonetheless it seems worth setting down our results briefly, so as to facilitate comparison with angle quantization.

Functions $\mathfrak{f}(r)$ of the radius cannot be written in product form as could functions of $p$ and $q$. The crucial step needed for quantization turns out to be consideration of the generating function for the orthonormal Hermite basis 
on $L^{2}(\mathbf{R})$, namely

$$
G_{t}(x)=\pi^{-1 / 4} e^{-t^{2} / 4+t x-x^{2} / 2}=\sum_{n \geq 0} t^{n} \frac{h_{n}(x)}{\sqrt{2^{n} n !}} .
$$

Substituting $\bar{G}_{s} \otimes G_{t}$ for $F$ in (2.2), we integrate over angles to get

$$
\begin{aligned}
\int_{-\pi}^{+\pi}\left[\mathscr{G}\left(\bar{G}_{s} \otimes G_{t}\right)\right](r \cos \beta, r \sin \beta) d \beta & =2 e^{-r^{2}} \sum_{n \geq 0} \frac{(-s t)^{n}}{2^{n} n !} L_{n}\left(2 r^{2}\right) \\
& =2 e^{-\frac{1}{2} s t} e^{-r^{2}} I_{0}(2 r \sqrt{s t})
\end{aligned}
$$

where $L_{n}$ is the $n$th Laguerre polynomial and $I_{0}$ is the modified Bessel function of the first kind, of order 0 .

Given any function of one variable, $\mathfrak{f} \in \mathcal{C}^{\infty}(\mathbf{R})$, use it to construct the phase space function $\tilde{\mathfrak{T}}_{\mathrm{rad}} \in \mathcal{C}^{(1)}(\Pi)$,

$$
\mathfrak{f}_{\mathrm{rad}}(p, q)=\mathfrak{f}\left(\sqrt{p^{2}+q^{2}}\right)
$$

$\mathfrak{f}_{\mathrm{rad}} \in \mathcal{C}^{\infty}(\Pi)$ is what we shall mean by a function of the radius alone. Consulting (2.1), we substitute $\mathfrak{f}_{\text {rad }}$ for $T$ and $\bar{G}_{s} \otimes G_{t}$ for $f \otimes g$. By using (2.11.a) and equating coefficients of identical powers of $s$ and $t$, we can evaluate $\Delta\left(\mathfrak{f}_{\text {rad }}\right)$ more or less explicitly.

As one might expect, the Hermite functions are eigenfunctions for $\Delta\left(\tilde{\mathfrak{f}}_{\text {rad }}\right)$ :

$$
\Delta\left(\mathfrak{f}_{\mathrm{rad}}\right) h_{n}=\rho_{n}(\mathfrak{f}) h_{n}, \quad n \geq 0,
$$

with eigenvalues

$$
\rho_{n}(\mathfrak{f})=(-1)^{n} \int_{0}^{\infty} \mathfrak{f}(\sqrt{u}) e^{-u} L_{n}(2 u) d u .
$$

As the Hermite functions are complete in $L^{2}(\mathbf{R})$, we can read off the spectral representation of $\Delta\left(\mathfrak{f}_{\mathrm{rad}}\right)$ :

$$
\Delta\left(\mathfrak{f}_{\mathrm{rad}}\right)=\sum_{n \geq 0} \rho_{n}(\mathfrak{f}) P_{n},
$$

where the $P_{n}$ are the orthogonal projections corresponding to the $h_{n}$.

It is standard that the Hermite functions $\left\{h_{n}: n \geq 0\right\}$ constitute a Schauder basis for $\mathscr{S}(\mathbf{R})$, and that the map

$$
\left(a_{n}\right)_{n \geq 0} \longrightarrow \sum_{n \geq 0} a_{n} h_{n}
$$

affords a topological isomorphism between the space of rapidly decreasing 
sequences and $\mathscr{S}(\mathbf{R})$ when both carry their usual topologies [4]. The dual of , can be identified with the space of polynomially bounded sequences.

Using estimates on the Laguerre functions together with $\mathfrak{f} \in \mathcal{C}^{(1)}(\mathbf{R})$, it may be shown that the sequence $\left(\rho_{n}(\mathfrak{f})\right)_{n \geq 0} \in J^{\prime}$, and so the above isomorphism between s and $\mathscr{S}(\mathbf{R})$ enables us to deduce that $\Delta\left(\mathfrak{f}_{\text {rad }}\right)$ is an element of $\mathscr{L}^{+}[\mathscr{S}(\mathbf{R})]$.

It is of particular interest to consider the quantization of the $k$ th power of the radius. Considered as a phase space function,

$$
r^{k}(p, q)=\left(p^{2}+q^{2}\right)^{k / 2}
$$

$r^{k} \in \mathbb{C}^{x}(\Pi)$. The integral for the eigenvalues may be done explicitly:

$$
\rho_{n}\left(r^{k}\right)=(-1)^{n} \Gamma\left(\frac{1}{2} k+1\right){ }_{2} F_{1}\left(-n, \frac{1}{2} k+1 ; 1 ; 2\right),
$$

in terms of the indicated hypergeometric function, which reduces to a polynomial here, cf [39]. In particular, the case $k=2$ is related to the number operator. Define the function

then

$$
v=\frac{1}{2}\left(r^{2}-1\right)
$$

$$
\Delta(v)=N
$$

A byproduct of these results is that we can find an integral relation involving the Moyal product of $r^{k}$ with $r^{l}$. Now the Moyal product $[17,19-22,29]$ is based on the following considerations. Let $R, S \in \mathscr{S}(\Pi)^{\prime}$ have quantizations $\Delta(R), \Delta(S)$, respectively, and suppose that the product $\Delta(R) \Delta(S)$ is well defined. Suppose further that there is a distribution $T$ such that

$$
\Delta(R) \Delta(S)=\Delta(T)
$$

Then we write

$$
T=R * S,
$$

and refer to $T$ as the Moyal or twisted product of $R$ and $S$. A symbolic expression for he Moyal product can be given which is certainly valid for distributions $R, S \in \mathscr{S}(\Pi) \subset \mathscr{S}(\Pi)^{\prime}$ :

$$
\begin{aligned}
& {[R * S]\left(p_{1}, q_{1}\right)} \\
& \quad=\left(1 / \pi^{2}\right) \int_{\Pi \times \Pi} R\left(p_{2}, q_{2}\right) S\left(p_{3}, q_{3}\right) \exp \left(-2 i\left|\begin{array}{ccc}
1 & 1 & 1 \\
p_{1} & p_{2} & p_{3} \\
q_{1} & q_{2} & q_{3}
\end{array}\right|\right) d p_{2} d q_{2} d p_{3} d q_{3} .
\end{aligned}
$$


In terms of the transform $\mathscr{G}$ of $(2.2)$,

$$
\left[\mathscr{G}^{-1}(R * S)\right](x, y)=(1 / 2 \pi) \int_{\mathbf{R}} \mathscr{G}^{-1}(R)(t, y) \mathscr{G}^{-1}(S)(x, t) d t .
$$

For the powers of the radius, it follows from (2.16) that

$$
\begin{gathered}
\Gamma\left(\frac{1}{2} k+1\right) \Gamma\left(\frac{1}{2} l+1\right) \quad{ }_{2} F_{1}\left(-n, \frac{1}{2} k+1 ; 1 ; 2\right){ }_{2} F_{1}\left(-n, \frac{1}{2} l+1 ; 1 ; 2\right) \\
=(-1)^{n} \int_{0}^{\infty}\left[r^{k} * r^{l}\right](\sqrt{u}) e^{-u} L_{n}(2 u) d u
\end{gathered}
$$

\section{§3. Quantization of Angle Functions}

Quantization of phase functions is technically more difficult than quantization of $p, q$ and $r$. This stems principally from the ambiguity in the definition of an angle function on phase space, viewed as a manifold. We must choose a branch of the arctangent and then define our angle function on all of $\Pi$ in a manner consistent with the branch chosen. To be precise, we shall take the angle function defined by the principal branch of the arctangent, for simplicity. Were we to choose another branch, an inessential reference angle $\theta$ would appear. Then

$$
\varphi(p, q)= \begin{cases}2 \arctan \left[\left(\sqrt{p^{2}+q^{2}}-p\right) / q\right], & \text { if } q \neq 0 \\ 0, & \text { if } q=0 \text { and } p \geq 0 \\ -\pi, & \text { if } q=0 \text { and } p<0\end{cases}
$$

Because of its importance, we have reserved the symbol $\varphi$ for this function.

Recall that the phase space function $v$ defined in (2.15.a) has the number operator as its quantization. Thus it is the natural candidate for a phase space function canonically conjugate to $\varphi$. A naive calculation [13] would suggest this, but in view of the branch cut in the definition of the phase angle, the Poisson bracket between $v$ and $\varphi$ must be defined as a distribution. By transferring the derivatives to the test function, we find that

$$
\{v, \varphi\}(F)=\int_{\Pi} \varphi(p, q)\left(q \frac{\partial F}{\partial p}-p \frac{\partial F}{\partial q}\right) d p d q, \quad F \in \mathscr{S}(\Pi) .
$$

This is justified because that part of the integrand multiplying $\varphi$ is seen to be in $\mathscr{S}(\Pi)$. The integral is meant in the Lebesgue sense, and can be done by methods similar to those for the kernel. We have given the necessary calculations in the appendix. The result is that 


$$
\{v, \varphi\}(p, q)=1+2 \pi p \chi_{(-\infty, 0)}(p) \delta(q) .
$$

The first term is canonical, and the additional term we shall call secular. As noted in the introduction, this secular term has a direct counterpart in the commutator of the number and phase operator (see Corollary 4.2 below):

$$
[N, \Delta(\varphi)] \neq i,
$$

which is, after all, where the problem began. It follows that to whatever extent the phase operator is observable, there must be experiments which will measure the effect of secular terms.

Along with the angle function we shall consider the functions $e^{ \pm i \varphi}$. As functions on phase space they must be defined so as to be consistent with $\varphi$. Thus, $e^{ \pm i \varphi}$ is given by

$$
e^{ \pm i \varphi}(p, q)= \begin{cases}(p \pm i q) /\left(p^{2}+q^{2}\right)^{1 / 2}, & \text { if } p^{2}+q^{2}>0 \\ 1, & \text { if } p=q=0\end{cases}
$$

As in the previous section we start our quantization procedure by substituting $\bar{G}_{s} \otimes G_{t}$ for $F$ in (2.2), but now we integrate over the radius. We then proceed as before, but with technical modifications. Starting with functions $\mathfrak{f} \in L^{2}([-\pi, \pi))$, we construct corresponding phase space functions $\mathfrak{f}_{\text {ang }} \in \mathcal{O}^{x}(\Pi)$, and substitute $\mathfrak{f}_{\text {ang }}$ for $T$ in (2.1). The integration over the radial variable leads to a complicated polynomial in the indices, first given in [39], cf also [32]. We have been able to simplify this considerably, expressing it as a ratio of gamma functions. In a sense the next lemma is the crux of our work, as the analysis of our proposed phase operator was our original motivation.

There is a certain amount of analysis necessary so as to avoid errors resulting from a careless treatment of singularities and distributions. For this reason, and because we do not know of any other treatment of this material, we have included many of the details.

Lemma 3.1. Substituting $\bar{G}_{s} \otimes G_{t}$ for $F$ in (2.2) and integrating over the radial variable, we obtain

$$
\int_{0}^{\infty}\left[\mathscr{G}\left(\overline{G_{s}} \otimes G_{t}\right)\right](r \cos \beta, r \sin \beta) r d r=\frac{1}{2 \pi} \sum_{m \cdot n \geq 0} i^{m-n} A_{m, n} s^{m} t^{n} e^{i(n-m) \beta}
$$

for all $s, t \in \mathbf{R}$ and $-\pi \leq \beta<\pi$, where

$$
A_{m, n}=\frac{2^{-\max (m . n)} \Gamma\left[\frac{1}{2} \min (m, n)+s(m, n)\right]}{\min (m, n) ! \Gamma\left[\frac{1}{2} \max (m, n)+s(m, n)\right]}, \quad m, n \geq 0 .
$$


We have introduced the convenient abbreviation

$$
s(m, n)= \begin{cases}1 / 2, & \min (m, n) \text { even } \\ 1, & \min (m, n) \text { odd }\end{cases}
$$

Proof. Doing a Gaussian integral and expanding the result as a Fourier series in the angle, we obtain

$$
\begin{aligned}
\int_{0}^{\infty}[ & \left.\mathscr{G}\left(\bar{G}_{s} \otimes G_{t}\right)\right](r \cos \beta, r \sin \beta) r d r \\
& =\frac{1}{\pi} e^{-\frac{1}{2} s t} \int_{0}^{\infty} e^{-r^{2}+i r\left(s e^{-\imath \beta}-t e^{\prime \beta}\right)} r d r \\
& =\frac{1}{2 \pi} \sum_{l, m . n \geq 0} \frac{i^{m-n} \Gamma\left(\frac{1}{2} m+\frac{1}{2} n+1\right)\left(-\frac{1}{2}\right)^{l}}{l ! m ! n !} s^{m+l} t^{n+l} e^{i(n-m) \beta} \\
& =\frac{1}{2 \pi} \sum_{m, n \geq 0} i^{m-n} A_{m . n} s^{m} t^{n} e^{i(n-m) \beta}
\end{aligned}
$$

with

$$
A_{m, n}=\sum_{l=0}^{\min (m, n)} \frac{\Gamma\left(\frac{1}{2} m+\frac{1}{2} n+1-l\right)}{l !(m-l) !(n-l) !}\left(-\frac{1}{2}\right)^{l} .
$$

We see from this that

$$
A_{m . n}=A_{n, m}, \quad m, n \geq 0
$$

and that

$$
A_{m, m}=\frac{1}{2^{m} m !}, \quad m \geq 0
$$

Let us fix $m$ and $n$ with $m<n$ until further notice, and write $s$ for $s(m, n)$. Our task is to simplity $A_{m, n}$.

$$
A_{m, n}=\frac{1}{m ! \Gamma\left(\frac{1}{2} n-\frac{1}{2} m\right)} \sum_{l=0}^{m}\left(\begin{array}{c}
m \\
l
\end{array}\right) B\left(\frac{1}{2} m+\frac{1}{2} n+1-l, \frac{1}{2} n-\frac{1}{2} m\right)\left(-\frac{1}{2}\right)^{l}
$$

where $B$ is the beta function,

$$
\begin{aligned}
B\left(\frac{x+1}{2}, \frac{y+1}{2}\right) & =\Gamma\left(\frac{x+1}{2}\right) \Gamma\left(\frac{y+1}{2}\right) / \Gamma\left(\frac{x+y}{2}+1\right) \\
& =2 \int_{0}^{\pi / 2} \sin ^{x} \beta \cos ^{y} \beta d \beta .
\end{aligned}
$$


Substituting the integral representation in the sum, we find

$$
\begin{aligned}
A_{m, n}= & \frac{1}{m ! 2^{n} \Gamma\left(\frac{1}{2} n-\frac{1}{2} m\right)} \int_{0}^{\pi} \sin ^{n-m-1} t\left(\cos ^{m} t+\cos ^{m+1} t\right) d t \\
= & \frac{1}{m ! 2^{n} \Gamma\left(\frac{1}{2} n-\frac{1}{2} m\right)} B\left(\frac{1}{2} m+s, \frac{1}{2} n-\frac{1}{2} m\right),
\end{aligned}
$$

from which the result is immediate.

With this result in hand, we are able to consider the matrix elements with respect to the Hermite basis.

Corollary 3.2. Let $\mathfrak{f} \in L^{2}([-\pi, \pi))$, and construct the phase space function

$$
\mathfrak{f}_{\text {ang }}(r \cos \beta, r \sin \beta)= \begin{cases}\mathfrak{f}(\beta) & \text { if } r>0, \\ 0 & \text { if } r=0,\end{cases}
$$

from it. Then $\tilde{\mathfrak{f}}_{\text {ang }} \in \mathcal{O}^{\infty}(\Pi)$, and

$$
\left[\Delta\left(\mathfrak{f}_{\text {ang }}\right) h_{n}\right]\left(\overline{h_{m}}\right)=\left[\frac{\max (m, n) ! 2^{\min (m, n)}}{\min (m, n) ! 2^{\max (m, n)}}\right]^{1 / 2} \frac{\Gamma\left(\frac{1}{2} \min (m, n)+s(m, n)\right)}{\Gamma\left(\frac{1}{2} \max (m, n)+s(m, n)\right)} i^{m-n} \hat{\mathfrak{f}}_{m-n},
$$

for all $m, n \geq 0$, where the

$$
\hat{\mathfrak{f}}_{k}=\frac{1}{2 \pi} \int_{-\pi}^{+\pi} \mathfrak{f}(\beta) e^{-i k \beta} d \beta
$$

are the Fourier coefficients of $\mathfrak{\mathfrak { i }}$.

Proof. We begin by showing that $\mathfrak{f}_{\text {ang }} \in \mathcal{O}^{\infty}(\Pi)$. As $\mathfrak{f} \in L^{2}([-\pi, \pi))$, the $\operatorname{map}$

$$
(p, q) \longrightarrow \frac{|\mathfrak{f}(\beta)|}{1+r^{2}} \in L^{2}(\Pi)
$$

Observing that

$$
\frac{|\mathfrak{f}(\beta)|}{\left(1+p^{2}\right)\left(1+q^{2}\right)} \leq \frac{|\mathfrak{f}(\beta)|}{1+r^{2}}
$$

the result follows.

By doing the radial integration first, we find that 


$$
\begin{aligned}
{\left[\Delta\left(\tilde{\mathfrak{f}}_{\text {ang }}\right) h_{n}\right]\left(\overline{h_{m}}\right) } & =\frac{A_{m, n}}{2 \pi} i^{m-n}\left(2^{n} n ! 2^{m} m !\right)^{1 / 2} \int_{-\pi}^{+\pi} \mathfrak{f}(\beta) e^{i(n-m) \beta} d \beta \\
& =A_{m, n} i^{m-n}\left(2^{m+n} m ! n !\right)^{1 / 2} \hat{\mathfrak{f}}_{m-n},
\end{aligned}
$$

from which the result follows upon expanding $A_{m . n}$.

In conjunction with this last result, we shall be able to retain $\mathcal{C}^{\prime x}(\Pi)$ as our symbol class for functions of the angle. We must now determine to which operator class $\Delta\left(\mathfrak{f}_{\text {ang }}\right)$ belongs when $\mathfrak{f}_{\text {ang }}$ is constructed as above. In order to do this we use the following estimate, obtained by using Stirling's formula.

Lemma 3.3. There exists a positive constant $K$ such that

$$
K^{-1}\left(\frac{\min (m, n)+1}{\max (m, n)+1}\right)^{1 / 4}\left|\hat{\mathfrak{f}}_{m-n}\right| \leq\left|\left[\Delta\left(\mathfrak{f}_{\text {ang }}\right) h_{n}\right]\left(\overline{h_{m}}\right)\right|
$$

and

$$
\left[\Delta\left(\mathfrak{f}_{\text {ang }}\right) h_{n}\right]\left(\overline{h_{m}}\right)\left|\leq K\left(\frac{\max (m, n)+1}{\min (m, n)+1}\right)^{1 / 4}\right| \hat{\mathfrak{f}}_{m-n} \mid
$$

for all $m, n \geq 0$.

Proof. Define the quantity

$$
\xi_{k: j}=2^{k / 2} \Gamma\left(\frac{k}{2}+j\right)(k !)^{-1 / 2},
$$

where $k \geq 0$ and $j=\frac{1}{2}$ or $j=1$. Using Stirling's approximation, we can deduce the existence of positive constants $A, B$ such that

$$
A(k+1)^{-1 / 4}<\xi_{k: \frac{1}{2}}<B(k+1)^{-1 / 4}
$$

and

$$
A(k+1)^{1 / 4}<\xi_{k ; 1}<B(k+1)^{1 / 4}
$$

Since

$$
\left|\left[\Delta\left(\tilde{\mathfrak{f}}_{\text {ang }}\right) h_{n}\right]\left(\overline{h_{m}}\right)\right|=\frac{\xi_{\min (m . n): s(m . n)}}{\xi_{\max (m . n): s(m . n)}}\left|\hat{\mathfrak{f}}_{m-n}\right|, \quad m, n \geq 0,
$$

the desired inequality follows with $K=B / A$.

This bound is basic to the next three results, which relate properties of $\mathfrak{f}_{\text {ang }}$ to the operator class of $\Delta\left(\mathfrak{f}_{\text {ang }}\right)$. Let $\mathfrak{f} \in L^{2}([-\pi, \pi))$ and let $\mathfrak{f}_{\text {ang }} \in \mathbb{C}^{x}(\Pi)$ be constructed as in Corollary 3.2. Let us introduce the abbreviation

$$
\hat{F}=\left(\hat{F}_{n}\right)_{n \in \mathbf{Z}}=\left((1+|n|)^{1 / 4}\left|\hat{\mathfrak{f}}_{n}\right|\right)_{n \in \mathbf{Z}}
$$


for the indicated sequence. We can characterize properties of the operator $\Delta\left(\mathfrak{f}_{\text {ang }}\right)$ in terms of properties of the sequence $\hat{F}$.

Proposition 3.4. $\Delta\left(\mathfrak{f}_{\text {ang }}\right) \in \mathscr{L}^{+}\left[\mathscr{S}(\mathbf{R}), L^{2}(\mathbf{R})\right]$ if and only if $\hat{F}$ is an element of $l^{2}(\mathbf{Z})$.

Proof. Suppose first that $\Delta\left(\mathfrak{f}_{\text {ang }}\right) \in \mathscr{L}^{+}\left[\mathscr{S}(\mathbf{R}), L^{2}(\mathbf{R})\right]$. Then for all $m \geq 0$,

$$
\begin{aligned}
\left|\left\langle h_{m}, \Delta\left(\mathfrak{f}_{\text {ang }}\right) h_{0}\right\rangle\right| & =\left|\left[\Delta\left(\mathfrak{f}_{\text {ang }}\right) h_{0}\right]\left(\overline{h_{m}}\right)\right| \\
& =\left(\xi_{0 ; \frac{1}{2}} / \xi_{m ; \frac{1}{2}}\right)\left|\hat{\mathfrak{f}}_{m}\right| \\
& \geq\left(\pi^{1 / 2} / B\right)(m+1)^{1 / 4}\left|\hat{\mathfrak{f}}_{m}\right| \\
& =\left(\pi^{1 / 2} / B\right) \hat{F}_{m} ;
\end{aligned}
$$

similarly,

$$
\left|\left\langle h_{m}, \Delta\left(\mathfrak{f}_{\text {ang }}\right)^{+} h_{0}\right\rangle\right| \geq\left(\pi^{1 / 2} / B\right) \hat{F}_{-m} .
$$

We deduce from this that $\hat{F} \in l^{2}(\mathbf{Z})$, with

$$
\sum_{m=-\infty}^{\infty}\left|\hat{F}_{m}\right|^{2} \leq\left(B^{2} / \pi\right)\left(\left\|\Delta\left(\mathfrak{f}_{\text {ang }}\right) h_{0}\right\|^{2}+\left\|\Delta\left(\mathfrak{f}_{\text {ang }}\right)^{+} h_{0}\right\|^{2}\right) .
$$

Conversely, suppose that $\hat{F} \in l^{2}(\mathbf{Z})$. Define $C>0$ by

$$
C^{2}=\sum_{n=-x}^{\infty}\left|\hat{F}_{n}\right|^{2}
$$

Since $\Delta\left(\mathfrak{f}_{\text {ang }}\right) \in \mathscr{L}\left[\mathscr{S}(\mathbf{R}), \mathscr{S}(\mathbf{R})^{\prime}\right]$, we notice that

$$
\begin{aligned}
\left|\left[\Delta\left(\tilde{f}_{\text {ang }}\right) h_{n}\right](\bar{\psi})\right| & =\left|\sum_{m \geq 0} \overline{\psi_{m}}\left[\Delta\left(\tilde{f}_{\text {ang }}\right) h_{n}\right]\left(\overline{h_{m}}\right)\right| \\
& \leq K \sum_{m \geq 0}\left|\psi_{m}\right|\left[\frac{\max (m, n)+1}{\min (m, n)+1}\right]^{1 / 4}\left|\hat{\tilde{f}}_{m-n}\right| \\
& \leq K \sum_{m \geq 0}\left|\psi_{m}\right|(|m-n|+1)^{1 / 4}\left|\hat{\tilde{f}}_{m-n}\right| \\
& =K \sum_{m \geq 0}\left|\psi_{m}\right| \hat{F}_{m-n} \\
& \leq K C\|\psi\|, \quad \psi \in \mathscr{S}(\mathbf{R}), n \geq 0
\end{aligned}
$$

where the norms are with respect to $L^{2}(\mathbf{R}), K$ is the constant appearing in the previous proposition, and

$$
\psi_{m}=\left\langle h_{m}, \psi\right\rangle .
$$


Thus $\Delta\left(\mathfrak{f}_{\text {ang }}\right) h_{n} \in L^{2}(\mathbf{R})$ with norm

$$
\left\|\Delta\left(\mathfrak{f}_{\text {ang }}\right) h_{n}\right\| \leq K C, \quad n \geq 0 .
$$

Introducing the family of seminorms $\left(p_{r}\right)_{r \geq 0}$ given by

$$
p_{r}(f)=\left\|(N+1)^{r} f\right\|, \quad f \in \mathscr{S}(\mathbf{R}), r \geq 0,
$$

which determine the usual topology on $\mathscr{S}(\mathbf{R})$, we see that $\Delta\left(\mathfrak{f}_{\text {ang }}\right) \in \mathscr{L}[\mathscr{S}(\mathbf{R})$, $\left.L^{2}(\mathbf{R})\right]$, and

$$
\begin{aligned}
\left\|\Delta\left(\mathfrak{f}_{\text {ang }}\right) \psi\right\| & \leq \sum_{n \geq 0}\left|\psi_{n}\right|\left\|\Delta\left(\mathfrak{f}_{\text {ang }}\right) h_{n}\right\| \\
& \leq K C \sum_{n \geq 0}\left|\psi_{n}\right| \\
& \leq \frac{\pi K C}{\sqrt{6}} p_{1}(\psi), \quad \psi \in \mathscr{S}(\mathbf{R}) .
\end{aligned}
$$

The $n$th Fourier component of the function $\overline{\mathfrak{f}}$ is the complex conjugate of $\hat{\mathfrak{f}}_{-n}$, so that the sequence $\left((1+|n|)^{1 / 4}\left|(\hat{\tilde{\mathfrak{f}}})_{n}\right|\right)_{n \in \mathbf{Z}}$ is in $l^{2}(\mathbf{Z})$. Then $\Delta\left(\overline{\mathfrak{f}}_{\text {ang }}\right) \in$ $\mathscr{L}\left[\mathscr{S}(\mathbf{R}), L^{2}(\mathbf{R})\right]$.

Now

$$
\overline{[\mathscr{G}(\bar{\varphi} \otimes \psi)(p, q)]}=\mathscr{G}(\bar{\psi} \otimes \varphi)(p, q), \quad \varphi, \psi \in \mathscr{S}(\mathbf{R}),(p, q) \in \Pi .
$$

Then

$$
\begin{aligned}
\left\langle\Delta\left(\overline{\mathfrak{f}}_{\text {ang }}\right) \varphi, \psi\right\rangle & =\overline{\left\langle\psi, \Delta\left(\overline{\mathfrak{f}}_{\text {ang }}\right) \varphi\right\rangle} \\
& =\overline{\overline{\mathfrak{f}}_{\text {ang }}[\mathscr{G}(\bar{\psi} \otimes \varphi)]} \\
& =\mathfrak{f}_{\text {ang }}[\mathscr{G}(\bar{\varphi} \otimes \psi)] \\
& =\left\langle\varphi, \Delta\left(\tilde{\mathfrak{f}}_{\text {ang }}\right) \psi\right\rangle, \quad \varphi, \psi \in \mathscr{S}(\mathbf{R}) .
\end{aligned}
$$

Hence $\Delta\left(\mathfrak{f}_{\text {ang }}\right) \in \mathscr{L}^{+}\left[\mathscr{S}(\mathbf{R}), L^{2}(\mathbf{R})\right]$, with

$$
\Delta\left(\tilde{f}_{\text {ang }}\right)^{+}=\Delta\left(\overline{\mathfrak{f}}_{\text {ang }}\right) .
$$

Proposition 3.5. A sufficient, but not necessary, condition that $\Delta\left(\mathfrak{f}_{\text {ang }}\right)$ be a bounded operator on $L^{2}(\mathbf{R})$ is that the Fourier coefficients of $\mathfrak{f}_{\text {ang }}$ be such that the sequence $\hat{F}$ is an element of $l^{1}(\mathbf{Z})$.

Proof. If $\hat{F} \in l^{1}(\mathbf{Z})$ then certainly $\hat{F} \in l^{2}(\mathbf{Z})$ and so $\Delta\left(\mathfrak{f}_{\text {ang }}\right) \in \mathscr{L}^{+}\left[\mathscr{S}(\mathbf{R}), L^{2}(\mathbf{R})\right]$. Then we can define a continuous function $F \in \mathscr{C}(\mathbf{T})$ by setting

$$
F\left(e^{i \beta}\right)=\sum_{n=-x}^{+\infty}\left|\hat{F}_{n}\right| e^{i n \beta}
$$


noting that

$$
\|F\|_{\infty} \leq \sum_{n=-\infty}^{\infty}\left|\hat{F}_{n}\right|
$$

so that the $n$th Fourier coefficient of $F$ is $\hat{F}_{n}$.

Let $\mathscr{H}$ be the Hilbert space

$$
\mathscr{H}=\left\{g \in L^{2}(\mathbf{T}): \hat{g}_{n}=0, n<0\right\},
$$

a closed subspace of $L^{2}(\mathbf{T})$. Let $P \in \mathscr{L}\left(L^{2}(\mathbf{T})\right)$ be the orthogonal projection onto $\mathscr{H}$. The function $F$ above serves to define a multiplication operator, a continuous linear operator $\mu(F) \in \mathscr{L}(\mathscr{H})$ by setting

$$
\mu(F)(g)=P(F g), \quad g \in \mathscr{H} .
$$

The symbol $F g$ stands for the function obtained by pointwise multiplication.

We note that

$$
\|\mu(F)(g)\|_{2}=\|P(F g)\|_{2} \leq\|F g\|_{2} \leq\|F\|_{\infty}\|g\|_{2}, \quad g \in \mathscr{H},
$$

so that

$$
\|\mu(F)\| \leq\|F\|_{\infty} \leq \sum_{n=-\infty}^{\infty}\left|\hat{F}_{n}\right|
$$

The map $\Phi: L^{2}(\mathbf{R}) \rightarrow \mathscr{H}$ given by

$$
\left(\Phi h_{n}\right)\left(e^{i \beta}\right)=e^{i n \beta}, \quad n \geq 0
$$

is an isometric isomorphism, and so we can transport $\mu(F)$ to $L^{2}(\mathbf{R})$ by setting

$$
A_{F}=\Phi^{-1} \circ \mu(F) \circ \Phi .
$$

As $\Phi$ is isometric, $A_{F} \in \mathscr{L}\left(L^{2}(\mathbf{R})\right)$, with

$$
\left\|A_{F}\right\|=\|\mu(F)\| \text {. }
$$

Let us calculate the matrix elements of $A_{F}$ :

$$
\begin{aligned}
\left\langle h_{m}, A_{F} h_{n}\right\rangle & =\left\langle\Phi h_{m}, \mu(F) \Phi h_{n}\right\rangle \\
& =\frac{1}{2 \pi} \int_{-\pi}^{\pi} F\left(e^{i \beta}\right) e^{i(n-m) \beta} d \beta \\
& =\hat{F}_{m-n}=(1+|m-n|)^{1 / 4}\left|\hat{\mathfrak{f}}_{m-n}\right|
\end{aligned}
$$

for all $m, n \geq 0$.

Using Lemma 3.3, we know that there exists a positive constant $K$ such that 


$$
\begin{aligned}
\left|\left\langle h_{m}, \Delta\left(\mathfrak{f}_{\text {ang }}\right) h_{n}\right\rangle\right| & \leq K(1+|m-n|)^{1 / 4}\left|\hat{\mathfrak{f}}_{m-n}\right| \\
& =K \hat{F}_{m-n}=K\left\langle h_{m}, A_{F} h_{n}\right\rangle
\end{aligned}
$$

for all $m, n \geq 0$.

For any function $\psi \in \mathscr{S}(\mathbf{R})$ with Hermite expansion $\sum_{n=0}^{\infty} \psi_{n} h_{n}$ we can define an associated function $|\psi| \in \mathscr{S}(\mathbf{R})$ by the expansion

$$
|\psi|=\sum_{n=0}^{\infty}\left|\psi_{n}\right| h_{n}
$$

Then

$$
\begin{aligned}
\left|\left\langle h_{m}, \Delta\left(\mathfrak{f}_{\text {ang }}\right) \psi\right\rangle\right| & \leq \sum_{n \geq 0}\left|\psi_{n}\right|\left|\left\langle h_{m}, \Delta\left(\mathfrak{f}_{\text {ang }}\right) h_{n}\right\rangle\right| \\
& \leq K \sum_{n \geq 0}\left|\psi_{n}\right|\left\langle h_{m}, A_{F} h_{n}\right\rangle \\
& =K\left\langle h_{m}, A_{F}|\psi|\right\rangle
\end{aligned}
$$

for all $m \geq 0$ and all $\psi \in \mathscr{S}(\mathbf{R})$.

Calculating the norm in $L^{2}(\mathbf{R})$,

$$
\begin{aligned}
\left\|\Delta\left(\mathfrak{f}_{\text {ang }}\right) \psi\right\|^{2} & =\sum_{m \geq 0}\left|\left\langle h_{m}, \Delta\left(\tilde{f}_{\text {ang }}\right) \psi\right\rangle\right|^{2} \\
& \leq K^{2} \sum_{m \geq 0}\left|\left\langle h_{m}, A_{F}|\psi|\right\rangle\right|^{2} \\
& =K^{2}\left\|A_{F}|\psi|\right\|^{2} \\
& \leq K^{2}\left\|A_{F}\right\|^{2}\|\psi\|^{2}
\end{aligned}
$$

for all $\psi \in \mathscr{S}(\mathbf{R})$, and so $\Delta\left(\mathfrak{f}_{\text {ang }}\right)$ is bounded, with

$$
\left\|\Delta\left(\mathfrak{f}_{\text {ang }}\right)\right\| \leq K\left\|A_{F}\right\| \leq K \sum_{n=-\infty}^{\infty}(1+|n|)^{1 / 4}\left|\hat{\mathfrak{f}}_{n}\right| .
$$

We shall show below that $\Delta(\varphi)$ does not satisfy this condition, but is bounded. Hence this condition is not necessary.

In the next proposition we give two necessary and sufficient conditions for $\Delta\left(\mathfrak{f}_{\text {ang }}\right) \in \mathscr{L}^{+}[\mathscr{S}(\mathbf{R})]$.

Proposition 3.6. The following three conditions are equivalent.

(i) $\Delta\left(\mathfrak{f}_{\text {ang }}\right) \in \mathscr{L}^{+}[\mathscr{S}(\mathbf{R})]$;

(ii) for any $k \geq 0$ there exists an integer $l \geq 0$ and a constant $C>0$, depending on $k$ but not $m$ and $n$, such that the Fourier coefficients of $\mathfrak{i}$ satisfy. 


$$
\left|\hat{\mathfrak{f}}_{m-n}\right|,\left|\hat{\mathfrak{f}}_{n-m}\right| \leq C \frac{(n+1)^{l}}{(m+1)^{k}}
$$

(iii) the Toeplitz matrix

$$
\tau\left(\mathfrak{f}_{\text {ang }}\right)=\left(\left|\hat{\mathfrak{f}}_{n-m}\right|\right)
$$

and its transpose, obtained from $\mathfrak{f}$, are elements of $\mathscr{L}^{+}(\jmath)$. In other words, the maps

$$
\left(a_{n}\right)_{n \geq 0} \longrightarrow\left(\sum_{m \geq 0}\left|\hat{\mathfrak{f}}_{n-m}\right| a_{m}\right)_{n \geq 0} \quad \text { and } \quad\left(a_{n}\right)_{n \geq 0} \longrightarrow\left(\sum_{m \geq 0}\left|\hat{\mathfrak{f}}_{m-n}\right| a_{m}\right)_{n \geq 0}
$$

are both linear endomorphisms of s.

Proof. (i) $\Rightarrow$ (ii) For any $k \geq 0$ we can find a positive constant $A$ and an index $l$ such that

$$
p_{k+1}\left(\Delta\left(\mathfrak{f}_{\text {ang }}\right) g\right) \leq A p_{l-1}(g)
$$

and

$$
p_{k+1}\left[\Delta\left(\mathfrak{f}_{\text {ang }}\right)^{+} g\right] \leq A p_{l-1}(g), \quad g \in \mathscr{S}(\mathbf{R}),
$$

where the seminorms $p_{k}$ were introduced in the previous Corollary. Then

$$
\begin{aligned}
(m+1)^{k}\left|\hat{\mathfrak{f}}_{m-n}\right| & \leq K(m+1)^{k+\frac{1}{4}}(n+1)^{\frac{1}{4}}\left|\left\langle\Delta\left(\mathfrak{f}_{\text {ang }}\right) h_{n}, h_{m}\right\rangle\right| \\
& \leq K(n+1)^{\frac{1}{4}} p_{k+1}\left(\Delta\left(\mathfrak{f}_{\text {ang }}\right) h_{n}\right) \\
& \leq K A(n+1)^{\frac{1}{4}} p_{l-1}\left(h_{n}\right) \\
& \leq K A(n+1)^{l-\frac{3}{4}} \\
& \leq K A(n+1)^{l} .
\end{aligned}
$$

The same argument works for $\hat{\mathfrak{f}}_{n-m}$ and $\Delta\left(\mathfrak{f}_{\text {ang }}\right)^{+}$.

(ii) $\Rightarrow$ (i) From the assumption,

$$
\begin{aligned}
(m+1)^{k}\left|\left[\Delta\left(\tilde{f}_{\text {ang }}\right) h_{n}\right]\left(h_{m}\right)\right| & \leq K(m+1)^{k+\frac{1}{4}}(n+1)^{\frac{1}{4}}\left|\hat{\mathfrak{f}}_{m-n}\right| \\
& \leq K(n+1)^{\frac{1}{4}}\left|\hat{\mathfrak{f}}_{m-n}\right| \\
& \leq K C(n+1)^{\frac{1}{4}}\left|\hat{\mathfrak{f}}_{m-n}\right| \\
& \leq K C(n+1)^{l-\frac{3}{4}} \\
& \leq K C(n+1)^{l} .
\end{aligned}
$$

Hence $\Delta\left(\mathfrak{f}_{\text {ang }}\right) \in \mathscr{L}[\mathscr{S}(\mathbf{R})]$, with 


$$
p_{k}\left(\Delta\left(\mathfrak{f}_{\text {ang }}\right) f\right) \leq K C p_{l}(f) .
$$

A similar argument holds for the adjoint, completing this implication. (ii) $\Leftrightarrow$ (iii) This is elementary, and so the proof is complete.

We now come to our candidate for a phase operator-the Weyl quantization of the angle function in phase space. We shall evaluate its Fourier coefficients and write down its matrix elements in detail. The matrix for $\Delta(\varphi)$ is far more complicated than the matrices for $P, Q$ and $\Delta\left(\mathfrak{f}_{\text {rad }}\right)$. These latter are either diagonal or non-zero only on the diagonals just above and below the main diagonal. The matrix for $\Delta(\varphi)$, in contrast, has no such support property. A careful examination, however, shows that alternate columns increase or decrease in tandem. We then show that $\Delta(\varphi) \in \mathscr{L}^{+}\left(\mathscr{S}(\mathbf{R}), L^{2}(\mathbf{R})\right]$, but $\Delta(\varphi) \notin \mathscr{L}^{+}[\mathscr{S}(\mathbf{R})]$. From the point of view either of operator theory or physics, $\Delta(\varphi)$ is far more interesting than the operators we previously considered.

Before writing down the matrix elements of $\Delta(\varphi)$ we should like to make a remark concerning the notation in respect of the angle. The phase space distribution $\varphi$ of $(3.1)$ is of the form $\mathfrak{f}_{\text {ang }}$, with the function $\mathfrak{f} \in L^{2}([-\pi, \pi))$ given by

$$
\mathfrak{f}(\beta)=\beta, \quad-\pi \leq \beta<\pi .
$$

In the context of this paper, the choice of the notation $\varphi$ for this $\mathfrak{f}_{\text {ang }}$ serves to emphasize that we are quantizing the angle function in phase space; the variable $\beta$ does not have this significance. However, this leaves open the notation for the corresponding function $\mathfrak{f}$, and we have decided to write $\mathfrak{f}=\varphi$. trusting that no misunderstanding will result from this abuse of notation. A similar convention holds for the phase space distributions $e^{ \pm i \varphi}$.

Proposition 3.7. The Fourier coefficients of $\varphi \in L^{2}([-\pi, \pi))$ are given by

$$
\hat{\varphi}_{k}=\frac{(-1)^{k+1}}{i k}\left(1-\delta_{k, 0}\right) \text {. }
$$

We conclude from this that the phase operator $\Delta(\varphi) \in \mathscr{L}^{+}\left[\mathscr{P}(\mathbf{R}), L^{2}(\mathbf{R})\right]$, but $\Delta(\varphi) \notin \mathscr{L}^{+}[\mathscr{S}(\mathbf{R})]$ and does not satisfy the condition of Proposition 3.5.

The matrix elements of the phase operator with respect to the Hermite basis are

$$
\begin{aligned}
& \left\langle h_{m}, \Delta(\varphi) h_{n}\right\rangle \\
& \quad=\left[1-\delta_{n, m}\right] \frac{i^{n-m-1}}{(n-m)} 2^{-|n-m| / 2}\left(\frac{\max (m, n) !}{\min (m, n) !}\right)^{1 / 2} \frac{\Gamma\left(\frac{1}{2} \min (m, n)+s(m, n)\right)}{\Gamma\left(\frac{1}{2} \max (m, n)+s(m, n)\right)} .
\end{aligned}
$$


Finally, we note that the phase operator is symmetric:

$$
\Delta(\varphi)^{+}=\Delta(\varphi)
$$

Proof. The Fourier coefficients result from evaluating the elementary integral

$$
\hat{\varphi}_{k}=\frac{1}{2 \pi} \int_{-\pi}^{+\pi} \beta e^{-i k \beta} d \beta
$$

They clearly satisfy the condition for $\Delta(\varphi) \in \mathscr{L}^{+}\left[\mathscr{S}(\mathbf{R}), L^{2}(\mathbf{R})\right]$. But if we consider $k=2$ in the second condition of the previous proposition, and let $n=0$, there is no $C$ such that

$$
\frac{1}{m} \leq \frac{C}{(m+1)^{2}}
$$

for all $m \geq 1$; hence $\Delta(\varphi) \notin \mathscr{L}^{+}[\mathscr{S}(\mathbf{R})]$. By comparing the sequence $\hat{F}$ with the harmonic sequence, we see that $\Delta(\varphi)$ does not satisfy the condition of Proposition 3.5.

The matrix elements of $\Delta(\varphi)$ between the Hermite functions follow from substituting the Fourier coefficients of $\varphi$ into (3.9). The symmetry is then evident from the symmetry of the matrix elements.

Were we to choose that branch of the arctangent determined by a cut from 0 to $\infty$ at the angle $\theta$, we would have to choose the angle function accordingly. With the same abuse of notation as for the angle function previously considered, we write $\varphi_{\theta}$ both for the phase space distribution $\mathfrak{f}_{\text {ang }}$ and the corresponding function $\mathfrak{f}$. Then

$$
\mathfrak{f}\left(e^{i \beta}\right)=\varphi_{\theta}\left(e^{i \beta}\right)=\beta \quad \theta \leq \beta<\theta+2 \pi .
$$

It is not very difficult to see that the matrix elements of the resulting phase operator are

$$
\left\langle h_{m}, \Delta\left(\varphi_{\theta}\right) h_{n}\right\rangle=(\theta+\pi) \delta_{n, m}+\left\langle h_{m}, \Delta(\varphi) h_{n}\right\rangle e^{i(n-m)(\theta+\pi)} .
$$

At this point we can contrast our phase operator with that found in references [6-8]. Notwithstanding the way these authors derived it, their result was the operator with matrix elements (up to a choice of $\theta$ )

$$
\left\langle h_{m}, \Delta(\Xi) h_{n}\right\rangle=\left[1-\delta_{n, m}\right] \frac{i^{m-n+1}}{n-m} .
$$

This is a bounded self adjoint operator, and the quantization of some phase space distribution $\Xi$. We do not have an explicit expression for $\Xi$, but are able nonetheless to prove that it is not a function of the angle (in phase space) alone. 
To see this, suppose $\Xi$ were of the form $\mathfrak{f}_{\text {ang }}$ for some function $\mathfrak{f} \in$ $L^{2}([-\pi, \pi))$. Then we would have

$$
\left(1-\delta_{m, n}\right) \frac{i^{m-n+1}}{n-m}=\frac{i^{m-n}}{2^{|m-n| / 2}}\left[\frac{\max (m, n) !}{\min (m, n) !}\right]^{1 / 2} \frac{\Gamma\left(\frac{1}{2} \min (m, n)+s(m, n)\right)}{\Gamma\left(\frac{1}{2} \max (m, n)+s(m, n)\right)} \hat{\mathfrak{f}}_{m-n} .
$$

We would then have

$$
\hat{\mathrm{f}}_{m}=-\left(1-\delta_{m .0}\right) \frac{2^{|m| / 2} i}{m} \frac{\Gamma\left(\frac{1}{2}|m|+\frac{1}{2}\right)}{(\pi|m| !)^{1 / 2}}
$$

for all $m \in \mathbf{Z}$. This can be substituted back into the previous equation and simplified, yielding the following identities:

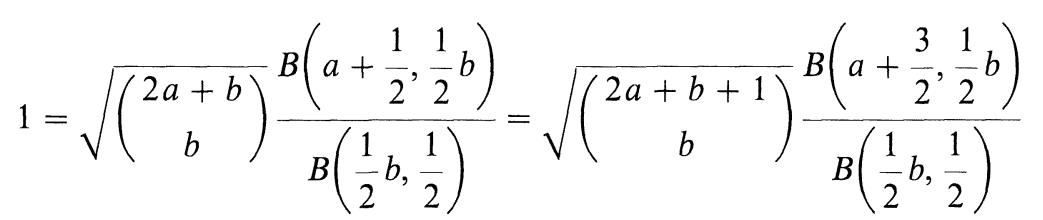

for all $a, b \geq 0$, where $B$ is the beta function. Using the reduction law for the beta function leads to

$$
\sqrt{\left(\begin{array}{c}
2 a+b \\
b
\end{array}\right)}=\frac{2 a+1}{2 a+b+1} \sqrt{\left(\begin{array}{c}
2 a+b+1 \\
b
\end{array}\right)}
$$

from which we deduce the requirement

$$
\frac{2 a+1}{2 a+b+1}=1
$$

for all $a, b \geq 0$, which is absurd. Thus $\Xi$ is not a function of the angle alone, which raises questions about its physical meaning as a phase operator.

We consider next the quantization of the phase space function $e^{i \varphi}$. In spite of its complicated definition, the matrix elements of $\Delta\left(e^{i \varphi}\right)$ are calculated by doing a very simple integral for the Fourier coefficients of $e^{i \varphi}$.

Corollary 3.8. The Fourier coefficients of $e^{i \varphi}$ are

$$
e^{i \varphi}=\delta_{1, k}
$$

Clearly $C=1$ and $l$ can be found so that condition (ii) of Proposition 3.6 is satisfied, implying that $\Delta\left(e^{i \varphi}\right) \in \mathscr{L}^{+}[\mathscr{S}(\mathbf{R})]$.

The matrix elements of $\Delta\left(e^{i \varphi}\right)$ are 


$$
\left\langle h_{m}, \Delta\left(e^{i \varphi}\right) h_{n}\right\rangle=i\left(\frac{n+1}{2}\right)^{\frac{1}{2}} \frac{\Gamma\left(\frac{1}{2} n+s_{n}\right)}{\Gamma\left(\frac{1}{2} n+s_{n}+\frac{1}{2}\right)} \delta_{m, n+1}
$$

where

$$
S_{n}= \begin{cases}\frac{1}{2}, & \text { if } n \text { is even } \\ 1, & \text { if } n \text { is odd }\end{cases}
$$

Thus $\Delta\left(e^{i \varphi}\right)$ is a weighted shift operator:

$$
\Delta\left(e^{i \varphi}\right) h_{n}=\lambda_{n+1} h_{n+1},
$$

where the weighting sequence $\lambda=\left(\lambda_{n}\right)_{n \geq 0}$ is given by'

$$
\lambda_{n+1}=i\left(\frac{n+1}{2}\right)^{\frac{1}{2}} \frac{\Gamma\left(\frac{1}{2} n+s_{n}\right)}{\Gamma\left(\frac{1}{2} n+s_{n}+\frac{1}{2}\right)}, \quad n \geq 0,
$$

and $\lambda_{0}=0$.

That is, the Weyl quantization of the phase space function $e^{i \varphi}$ determines a particular solution $\Delta\left(e^{i \varphi}\right)=U_{i}^{*}$ of the commutation relations

$$
\left[U_{i}^{*}, N\right]_{-}=-U_{i}^{*} \text {. }
$$

We note the obvious fact that $U_{i}$ is the quantization of the function $e^{-i \varphi}$.

The sequence $\lambda$ given in (3.17.b) does not satisfy the conditions of Lerner et al [10]. Rather, the even and odd indexed subsequences monotonically approach unity from below and above, respectively. It is possible to determine the spectral properties of $U_{\lambda}$ and its adjoint.

Proposition 3.9. Fixing the sequence $\lambda$ as in (3.17), we drop it as a subscript, writing $U$ for $\Delta\left(e^{-i \varphi}\right)$ and $U^{*}$ for $\Delta\left(e^{i \varphi}\right)$. Then $U^{*}$ maps $\mathscr{S}(\mathbf{R})$ into itself continuously. It is bounded, with

$$
\left\|U^{*}\right\|=(\pi / 2)^{1 / 2},
$$

and spectral radius

$$
r_{\sigma}\left(U^{*}\right)=1 .
$$

Its spectrum is 


$$
\sigma\left(U^{*}\right)=\overline{\mathbf{D}}
$$

where $\mathbf{D}$ is the open unit disc in $\mathbf{C}$ and

$$
\overline{\mathbf{D}}=\{z \in \mathbf{C}:|z| \leq 1\}
$$

the closed unit disc.

The spectrum of $U^{*}$ contains no eigenvalues; its residual spectrum is the open unit disc and its boundary is the continuous spectrum:

$$
\sigma_{\mathrm{pt}}\left(U^{*}\right)=\varnothing, \sigma_{\mathrm{res}}\left(U^{*}\right)=\mathbf{D}, \sigma_{\text {cont }}\left(U^{*}\right)=\overline{\mathbf{D}} \backslash \mathbf{D} .
$$

The operator $U$ maps $\mathscr{S}(\mathbf{R})$ into itself continuously, is bounded, with norm

$$
\|U\|=(\pi / 2)^{1 / 2}
$$

and spectral radius

$$
r_{\sigma}(U)=1
$$

Its spectrum is

$$
\sigma(U)=\overline{\mathbf{D}}
$$

The residual spectrum is empty, the continuous spectrum is the boundary of the unit disc, and all the points of the open unit disc are eigenvalues:

$$
\sigma_{\mathrm{pt}}(U)=\mathbf{D}, \sigma_{\text {res }}(U)=\varnothing, \sigma_{\text {cont }}(U)=\overline{\mathbf{D}} \backslash \mathbf{D} \text {. }
$$

Each eigenvalue $z \in \mathbf{D}$ of $U$ is of unit multiplicity, with eigenvector

$$
e_{z}=h_{0}+\sum_{n=1}^{\infty}\left(\prod_{k=1}^{n} \lambda_{k}\right)^{-1}(-z)^{n} h_{n}
$$

and the family $\left\{e_{z}: z \in \mathbf{D}\right\}$ forms an overcomplete set.

Proof. That these operators are bounded and map $\mathscr{S}(\mathbf{R})$ into itself continuously follows from our work above. The remainder of the proof is very much as in [10], but without the chain condition and taking into account that our sequence $\lambda$ is not monotonic.

The definitions of $U$ and $U^{*}$ yield their norms in short order. The spectral radius of $U^{*}$ is found by considering

$$
\left(U^{*}\right)^{k} h_{n}=i^{k} \mu(k ; n) h_{k+n},
$$

where 


$$
\mu(k ; n)=\prod_{r=n+1}^{n+k}\left|\lambda_{r}\right|
$$

so that

$$
\left\|\left(U^{*}\right)^{k}\right\|=\sup _{n}|\mu(k ; n)|
$$

Although the alternate elements of $\lambda$ straddle unity, the sequences

$$
n \longrightarrow\left|\lambda_{2 n+1}\right| \text { and } n \longrightarrow\left|\lambda_{2 n+1} \lambda_{2 n+2}\right|
$$

are decreasing. Hence

$$
\mu(2 k ; 2 n)>\mu(2 k ; 2 n+1) \text { and } \mu(2 k ; 2 n)>\mu(2 k ; 2 n+2),
$$

so

$$
\left\|\left(U^{*}\right)^{2 k}\right\|=\mu(2 k ; 0) .
$$

Moreover, using Cesàro convergence,

$$
r_{\sigma}\left(U^{*}\right)=\lim _{k \rightarrow \infty} \mu(2 k ; 0)^{1 / 2 k}=\lim _{k \rightarrow \infty}\left[\prod_{r=1}^{2 k}\left|\lambda_{r}\right|\right]^{1 / 2 k}=1 ;
$$

this is also the spectral radius of $U$ on general principles.

Writing

$$
e_{z}=\sum_{n \geq 0} \xi_{n}(z) h_{n}
$$

the ratio test shows immediately that $e_{z} \in L^{2}(\mathbf{R})$ for all $z \in \mathbf{D}$. Direct calculation shows that $e_{z}$ is an eigenvector of $U$ with eigenvalue $z$, and that $z$ is a nondegenerate eigenvalue of $U$.

For $z \in \overline{\mathbf{D}} \backslash \mathbf{D}$ the ratio test fails to give a result. However, Stirling's approximation shows that

$$
\lim _{n \rightarrow \infty}\left|n\left(\left|\frac{\xi_{n}(z)}{\xi_{n+1}(z)}\right|^{2}-1\right)\right|=\lim _{n \rightarrow \infty}\left|n\left(\left|\lambda_{n+1}\right|^{2}-1\right)\right|=\frac{1}{2} .
$$

Then we can find a positive integer $N$ such that

$$
n\left(\left|\frac{\xi_{n}(z)}{\xi_{n+1}(z)}\right|^{2}-1\right) \leq 3 / 4
$$

for all $n \geq N$. Hence Raabe's test tells us that $\left[\xi_{n}(z)\right] \notin l^{2}$, so we cannot define $e_{z} \in L^{2}(\mathbf{R})$ for $z \in \overline{\mathbf{D}} \backslash \mathbf{D}$. Thus we deduce that

$$
\sigma_{\mathrm{pt}}(U)=\mathbf{D} \subset \sigma(U) \subset \overline{\mathbf{D}},
$$


and so

$$
\sigma(U)=\sigma\left(U^{*}\right)=\overline{\mathbf{D}}
$$

Finally, a simple calculation shows that $U^{*}$ has no eigenvalues, so its point spectrum is empty. This implies that the residual spectrum of $U$ is empty, and the residual spectrum of $U^{*}$ is $\mathbf{D}$. This completes the proof.

This result is more or less remarkable, as we did not set out to choose a solution of (3.18) in the sense of [10] or a variation of those results. Rather, employing first principles, a physically motivated choice led to an analogous result. It seems worth re-emphasizing that $\Delta\left(e^{i \varphi}\right)$ and $\Delta\left(e^{-i \varphi}\right)$ are not the partial isometries corresponding to the raising and lowering operators, nor do they satisfy the monotonic chain condition. The even-odd index interleaving that occurs seems quite interesting mathematically, and is further evidence, if any is needed, that these operators must be handled with care.

\section{§4. Kernels, Commutators and Expectations}

So far, we have determined the operators of quantization in terms of their matrix elements. For certain purposes it is useful to know the kernels of operators, and we shall begin this section by determining the kernels of $\Delta(\varphi)$ and $\Delta\left(e^{i \varphi}\right)$.

There is no great difficulty in finding a formal expression for the kernel of $\Delta(\varphi)$; the difficulty lies in justifying it. To do so in detail has required a rather elaborate exercise in integration theory, not very illuminating in itself, but there does not seem to be any reliable means to avoid it. We have placed this analysis in an appendix. The result is contained in Proposition A.14: for every $f, g \in \mathscr{S}(\mathbf{R})$ we have

$$
\begin{aligned}
\langle f, \Delta(\varphi) g\rangle= & \frac{\pi}{2} \int_{\mathbf{R}} \operatorname{sign}(q) \overline{f(q)} g(q) d q \\
& -\frac{i}{2} \lim _{L \rightarrow \infty} \int_{\mathbf{R}^{2}} \operatorname{sign}(y) e^{-|x y|} g_{I(L)}(x) \overline{\left(y+\frac{1}{2} x\right)} g\left(y-\frac{1}{2} x\right) d x d y,
\end{aligned}
$$

where the integrals are meant in the Lebesgue sense, where

$$
g_{I(L)}(x)= \begin{cases}x^{-1}, & \text { if }|x| \in\left[L^{-1}, L\right] \\ 0, & \text { otherwise }\end{cases}
$$

and 


$$
\operatorname{sign}(t)= \begin{cases}t /|t|, & \text { if } t \in \mathbf{R} \backslash\{0\} \\ 0, & \text { if } t=0\end{cases}
$$

These expressions define a tempered distribution $\mathscr{K}_{\Delta(\varphi)} \in \mathscr{S}\left(\mathbf{R}^{2}\right)^{\prime}$ by

$$
\langle f, \Delta(\varphi) g\rangle=\mathscr{K}_{\Delta(\varphi)}(\bar{f} \otimes g),
$$

and this distribution is what is meant by the kernel of the operator $\Delta(\varphi)$.

Now there is no ambiguity about the definition of the kernel, but it is usual for physicists to write down an expression for a kernel as a singular or generalized function. Provided that such an expression is understood to be a symbolic shorthand for (4.1) and (4.2), this is unobjectionable. To arrive at such an expression, we must substitute the expression for $g_{I(L)}$ and $F$ into (4.1.b), throw all caution to the winds and interchange limit and integral, write

$$
\langle f, \Delta(\varphi) g\rangle=\int_{\mathbf{R}^{2}} \mathscr{K}_{\Delta(\varphi)}(x, y) \overline{f(x)} g(y) d x d y,
$$

and then

$$
\mathscr{K}_{\Delta(\varphi)}(x, y)=\frac{1}{2} \pi \operatorname{sign}(x+y) \delta(x-y)+\frac{1}{2 i} \operatorname{sign}(x+y) e^{-\frac{1}{2}\left|x^{2}-y^{2}\right|} \frac{1}{(x-y)} .
$$

We note that the kernel is conjugate symmetric in $x$ and $y$, as we would expect.

As the Fourier transform is a continuous isomorphism of $\mathscr{S}\left(\mathbf{R}^{2}\right)^{\prime}$ we can proceed in the same way for the tempered distribution $\mathscr{F}_{\Delta(\varphi)}$. We find that

$$
\mathscr{F} \mathscr{K}_{\Delta(\varphi)}(x, y)=\frac{1}{2 i}\left(1+\operatorname{sign}(y-x)-\operatorname{sign}(y-x) e^{-\frac{1}{2}\left|x^{2}-y^{2}\right|}\right) \frac{1}{(x+y)},
$$

with the same proviso about its meaning. The high degree of similarity between $\mathscr{K}_{\Delta(\varphi)}$ and $\mathscr{F}_{\boldsymbol{K}_{(\varphi)}}$ is intriguing, and follows since

$$
\mathscr{G}(\bar{f} \otimes g)(p, q)=\mathscr{G}(\overline{\mathscr{F} f} \otimes \mathscr{F} g)(-q, p), \quad f, g \in \mathscr{S}(\mathbf{R}),(p, q) \in \Pi,
$$

so that

$$
\begin{aligned}
{\left[\mathscr{F}_{\Delta(\varphi)}\right](\overline{\mathscr{R} f} \otimes g) } & =\mathscr{K}_{\Delta(\varphi)}(\mathscr{F}[\overline{\mathscr{R} f} \otimes g]) \\
& =\mathscr{K}_{\Delta(\varphi)}(\overline{\mathscr{F} f} \otimes \mathscr{F} g) \\
& =\langle\mathscr{F} f, \Delta(\varphi) \mathscr{F} g\rangle \\
& =\int_{\Pi} \varphi(-q, p) \mathscr{G}(\bar{f} \otimes g)(p, q) d p d q,
\end{aligned}
$$


for all $f, g \in \mathscr{S}(\mathbf{R})$, and where $\mathscr{R} \in \mathscr{L}\left[L^{2}(\mathbf{R})\right]$ is given by

$$
\mathscr{R} f(x)=f(-x), \quad f \in L^{2}(\mathbf{R}) .
$$

The distribution $(p, q) \rightarrow \varphi(-q, p)$, which is well defined, is simply the distribution $\varphi$ rotated through an angle $\pi / 2$ in phase space [29]. Then

$$
\varphi(-q, p)=\varphi(p, q)+\frac{1}{2} \pi-2 \pi \chi_{(-\infty .0)}(p) \quad \chi_{(0 . \infty)}(q), \quad(p, q) \in \Pi .
$$

Thus $\mathscr{F}_{\boldsymbol{K}_{(\varphi)}}(\overline{\mathscr{R} f} \otimes g)$ and $\mathscr{K}_{\Delta(\varphi)}(\bar{f} \otimes g)$ are indeed closely related.

The kernel of any operator $\Delta(T)$ must be defined with the same care we gave $\Delta(\varphi)$. We have done so for $\Delta\left(e^{i \varphi}\right)$ and $\Delta\left(r^{2}\right)$, but will omit the tedious details. We have found that

$$
\begin{aligned}
& \mathscr{K}_{\Delta\left(e^{i \varphi}\right)}(x, y) \\
& \quad=\frac{i}{2 \pi}(x+y)\left[K_{0}\left(\frac{1}{2}\left|x^{2}-y^{2}\right|\right)+\pi^{1 / 2} \operatorname{sign}\left(x^{2}-y^{2}\right) K_{1}\left(\frac{1}{2}\left|x^{2}-y^{2}\right|\right)\right],
\end{aligned}
$$

where $K_{n}$ is the modified Bessel function of the second kind of index $n$. For the square of the radius we find that

$$
\mathscr{K}_{\Delta\left(r^{2}\right)}(x, y)=\left(\frac{x+y}{2}\right)^{2} \delta(x-y)-\delta^{\prime \prime}(x-y) .
$$

This completes our treatment of kernels.

We observed at the outset that there could be no canonical phase operator. Now that we have a phase operator, it is natural to enquire what its (non-canonical) commutation relation with the number operator is. Because the phase operator maps $\mathscr{S}(\mathbf{R})$ into $L^{2}(\mathbf{R})$, and the number operator is unbounded, a commutator of $N$ and $\Delta(\varphi)$ cannot be defined as an operator on Hilbert space.

This problem occurs for all operators which map $\mathscr{S}(\mathbf{R})$ into $L^{2}(\mathbf{R})$, and we propose defining the commutator between all such operators as forms [31]; we shall call these forms generalized commutators. Using the theory of forms, a representation of a generalized commutator as a map in $\mathscr{L}\left[\mathscr{S}(\mathbf{R}), \mathscr{S}(\mathbf{R})^{\prime}\right]$ is possible. But then a generalized commutator must be the quantization of a tempered distribution. Our definition is consistent in that if both operators from which the generalized commutator is constructed happen to belong to $\mathscr{L}^{+}[\mathscr{S}(\mathbf{R})]$, the representing map in $\mathscr{L}\left[\mathscr{S}(\mathbf{R}), \mathscr{S}(\mathbf{R})^{\prime}\right]$ is, in fact, an element of $\mathscr{L}^{+}[\mathscr{S}(\mathbf{R})]$.

Proposition 4.1. Let $A, B \in \mathscr{L}^{+}\left[\mathscr{S}(\mathbf{R}), L^{2}(\mathbf{R})\right] . \quad$ By the generalized commu- 
tator of $A$ and $B$ we mean the sesquilinear form

$$
\tau_{A, B}: \mathscr{S}(\mathbf{R}) \times \mathscr{S}(\mathbf{R}) \longrightarrow \mathbf{C}
$$

given by

$$
\tau_{A, B}[f, g]=\left\langle A^{+} f, B g\right\rangle-\left\langle B^{+} f, A g\right\rangle, \quad f, g \in \mathscr{S}(\mathbf{R}) .
$$

Given $\tau_{A, B}$, there exists an $X_{A, B} \in \mathscr{L}\left[\mathscr{S}(\mathbf{R}), \mathscr{S}(\mathbf{R})^{\prime}\right]$ such that

$$
\tau_{A, B}[f, g]=\left[X_{A, B} g\right](\bar{f}), \quad f, g \in \mathscr{S}(\mathbf{R}),
$$

where

$$
X_{A, B}=\widehat{A^{+}} B-\widehat{B^{+}} A .
$$

We note that as $X_{A, B} \in \mathscr{L}\left[\mathscr{S}(\mathbf{R}), \mathscr{S}(\mathbf{R})^{\prime}\right]$ there exists a distribution $T_{A, B} \in \mathscr{S}\left(\mathbf{R}^{2}\right)^{\prime}$ such that

$$
X_{A, B}=\Delta\left(T_{A, B}\right) .
$$

By $\hat{A} \in \mathscr{L}\left[L^{2}(\mathbf{R}), \mathscr{S}(\mathbf{R})^{\prime}\right]$ we mean the continuous operator defined by

$$
[\hat{A}(g)](f)=\langle A(\bar{f}), g\rangle, \quad A \in \mathscr{L}^{+}[\mathscr{S}(\mathbf{R})], f \in \mathscr{S}(\mathbf{R}), g \in L^{2}(\mathbf{R}) .
$$

If the operators $A, B \in \mathscr{L}^{+}[\mathscr{S}(\mathbf{R})]$, then

$$
X_{A, B}=[A, B]_{-},
$$

which belongs to $\mathscr{L}^{+}[\mathscr{S}(\mathbf{R})]$.

Proof. The only non-trivial part of the proof is the continuity of $\hat{A}$ for any $A \in \mathscr{L}^{+}\left[\mathscr{S}(\mathbf{R}), L^{2}(\mathbf{R})\right]$. As $\hat{A}$ differs from the adjoint of $A$ only by the incorporation of some necessary conjugations, this is straightforward to show, and we omit it.

We note that, in general, these generalized commutator forms can be extended, the extension depending on the particular operators involved. We shall not consider this here.

Of particular interest is the commutator between the phase and number operators.

Corollary 4.2. The matrix elements of the generalized commutator of the phase and number operators with respect to the Hermite basis are $\tau_{N . \Delta(\varphi)}\left[h_{m}, h_{n}\right]=(m-n)\left\langle h_{m}, \Delta(\varphi) h_{n}\right\rangle$ 


$$
=-\left[1-\delta_{n, m}\right] i^{n-m-1} 2^{-! n-m ! / 2}\left(\frac{\max (m, n) !}{\min (m, n) !}\right)^{1 / 2} \frac{\Gamma\left(\frac{1}{2} \min (m, n)+s(m, n)\right)}{\Gamma\left(\frac{1}{2} \max (m, n)+s(m, n)\right)},
$$

for all $n, m \geq 0$.

The tempered distribution on phase space whose quantization is this generalized commutator is

$$
T_{N, \Delta(\varphi)}(F)=2 \pi i \int_{0}^{\infty} F(-r, 0) r d r-i \int_{\Pi} F(p, q) d p d q, \quad \forall F \in \mathscr{S}(\Pi) .
$$

Let $U \in \mathscr{L}\left[\mathscr{S}(\mathbf{R}), \mathscr{S}(\mathbf{R})^{\prime}\right]$ be defined by

$$
[U g](f)=\lim _{L \rightarrow \infty} \int_{\mathbf{R}} g_{I(L)}(x) f(x) g(-x) d x, \quad f, g \in \mathscr{S}(\mathbf{R}) .
$$

Then the map $X_{N, \Delta(\varphi)} \in \mathscr{L}\left[\mathscr{S}(\mathbf{R}), \mathscr{S}(\mathbf{R})^{\prime}\right]$ which is the quantization of the distribution $T_{N, \Delta(\varphi)}$ is given by

$$
\begin{aligned}
{\left[X_{N, \Delta(\varphi)} g\right](f)=} & -i g(f)+\frac{1}{2} i\left(-[U g]\left(f^{\prime}\right)+\left[U g^{\prime}\right](f)\right) \\
& +\frac{1}{2} \pi\left[g(0) f^{\prime}(0)-g^{\prime}(0) f(0)\right], \quad f, g \in \mathscr{S}(\mathbf{R}),
\end{aligned}
$$

Symbolically, we can write this as

$$
X_{N, \Delta(\varphi)} g=-i g+\frac{1}{2} i\left[(U g)^{\prime}+U g^{\prime}\right]-\frac{1}{2} \pi\left[g(0) \delta^{\prime}+g^{\prime}(0) \delta\right], \quad g \in \mathscr{S}(\mathbf{R}),
$$

where $\delta$ is the delta distribution concentrated at the origin and the primes indicate derivatives in the distributional sense.

Proof. The matrix elements may be read off from the general expression. We can also find the matrix element between Hermite generating functions. In a notation we used for finding the matrix elements of general functions of the angle,

$$
\begin{aligned}
& {\left[X_{N, \Delta(\varphi)} G_{t}\right]\left(\bar{G}_{s}\right)} \\
& \quad=\tau_{N, \Delta(\varphi)}\left[G_{s}, G_{t}\right] \\
& \quad=i \sum_{n, m \geq 0} i^{m-n} A_{m, n} s^{m} t^{n}\left[(-1)^{m-n}-\delta_{m . n}\right]
\end{aligned}
$$




$$
\begin{aligned}
& =2 \pi i \int_{0}^{\infty}\left[\mathscr{G}\left(\bar{G}_{s} \otimes G_{t}\right)\right](-r, 0) r d r-i e^{\frac{1}{2} s t} \\
& =2 \pi i \int_{0}^{\infty}\left[\mathscr{G}\left(\bar{G}_{s} \otimes G_{t}\right)\right](-r, 0) r d r-i \int_{\Pi}\left[\mathscr{G}\left(\bar{G}_{s} \otimes G_{t}\right)\right](p, q) d p d q
\end{aligned}
$$

for all real $s, t$. With the definition of $T_{N, \Delta(\varphi)}$ above, this yields

$$
X_{N, \Delta(\varphi)}=\Delta\left(T_{N, \Delta(\varphi)}\right) .
$$

We shall not write down the derivation of (4.14.b); the techniques are those of the appendix. We do note, however, that

$$
2 \pi i r[\mathscr{G}(f \otimes g)](-r, 0)=\sqrt{2 \pi}\left[\mathscr{F} h^{\prime}\right](2 r),
$$

where

$$
h(x)=f(x) g(-x)
$$

which leads to

$$
\begin{aligned}
{\left[X_{N, \Delta(\varphi)} g\right](f)=} & \frac{1}{2} \sqrt{\frac{\pi}{2}} \int_{\mathbf{R}}[1+\operatorname{sign}(r)]\left[\mathscr{F} h^{\prime}\right](r) d r-i \int_{\mathbf{R}} f(x) g(x) d x \\
= & \frac{\pi}{2}\left[f^{\prime}(0) g(0)-f(0) g^{\prime}(0)\right]-i \int_{\mathbf{R}} f(x) g(x) d x \\
& -\frac{i}{2} \lim _{L \rightarrow \infty} \int_{\mathbf{R}} g_{I(L)}(x) h^{\prime}(x) d x
\end{aligned}
$$

from which the result follows in a few steps.

Equation (4.14) displays the generalized commutator of $N$ and $\Delta(\varphi)$ in a form in which the deviation from canonicity is explicit. We shall refer to $-i g$ as the canonical term, and the other terms as secular. It is the presence of these terms which makes working with $\Delta(\varphi)$ so delicate; it is to avoid these terms that the $C$ and $S$ operators in the literature have been constructed.

We note that if we were to change units so that $\hbar$ were to appear explicitly, then the secular terms would be multiplied by a factor $\hbar$. We conclude this by dimensional arguments: the secular terms differ from the canonical term by a dimensional factor length ${ }^{-2}$.

This leaves an interesting problem. If we were able to exponentiate $\Delta(\varphi)$, the exponential would differ from $\Delta\left(e^{i \varphi}\right)$ by the secular terms. It would also differ from any of the $U_{\lambda}^{*}$ operators so far considered in the literature. As these secular effects are of higher order in $\hbar$ than the canonical term, one 
suspects that all these "exponentials" will have the same classical limit. But the secular effects are of crucial importance for the theoretical and structural considerations of quantum mechanical phase. It is only by operational considerations that a final choice of phase operator can be made. Perhaps more than one sort of phase phenomenon occurs, and one requires different operators for these different effects. It is tempting to conjecture that the quantum optical effects described by the phase operator requires $\Delta(\varphi)$, and the analysis in the literature has left out higher order quantum effects.

As well as commutators, another construct of importance in quantum theory is the expectation value of an operator in a given state. As we are mostly considering maps in $\mathscr{L}^{+}\left[\mathscr{S}(\mathbf{R}), L^{2}(\mathbf{R})\right]$, the existence of expectations requires delicate arguments which depend upon the particular operator being considered. For the expectation of the square of such a map, things are even more delicate. In particular, we shall have to give a definition of expectation which gives meaning to the relevant trace.

In the remainder of this section we shall consider only functions $\mathfrak{i} \in$ $L^{2}([-\pi, \pi))$ and the corresponding phase space function $\mathfrak{f}_{\text {ang }} \in \mathcal{C}^{\infty}(\Pi)$ of the angle alone, cf, Corollary 3.2. We shall suppose that $\mathfrak{f}$ satisfies the condition of Proposition 3.4 and is real valued, so that $\Delta\left(\mathfrak{f}_{\text {ang }}\right) \in \mathscr{L}^{+}\left[\mathscr{S}(\mathbf{R}), L^{2}(\mathbf{R})\right]$ is symmetric. Of course this includes the phase operator.

Proposition 4.3. Let $\mathfrak{f}$ be as stated above. Then

$$
\operatorname{tr}\left[\overline{\left(X^{+}\right)^{*} \Delta\left(\mathfrak{f}_{\text {ang }}\right)}\right]=\operatorname{tr}\left[\overline{\Delta\left(\mathfrak{f}_{\text {ang }}\right) X}\right], \quad X \in \mathscr{L}^{+}[\mathscr{S}(\mathbf{R})]^{\prime} ;
$$

and we refer to the common value

$$
\operatorname{Exp}\left[\Delta\left(\mathfrak{f}_{\text {ang }}\right) ; X\right]=\operatorname{tr}\left[\overline{\Delta\left(\mathfrak{f}_{\text {ang }}\right) X}\right]
$$

as the expectation of the operator $\Delta\left(\mathfrak{f}_{\text {ang }}\right)$ in the state $X \in \mathscr{L}^{+}[\mathscr{S}(\mathbf{R})]^{\prime}$, where by a state we mean a positive normalized element of $\mathscr{L}^{+}[\mathscr{S}(\mathbf{R})]^{\prime}$.

Proof. Since $\mathfrak{f}$ satisfies the conditions of Proposition 3.4, the sequence $\hat{F} \in l^{2}(\mathbf{Z})$, and so we can define a function $F \in L^{2}([-\pi, \pi))$ by setting

$$
F\left(e^{i \beta}\right)=\sum_{n=-x}^{\infty} \hat{F}_{n} e^{i n \beta}
$$

We also define $g \in L^{x}([-\pi, \pi))$ by setting

$$
g\left(e^{i \beta}\right)=\sum_{k=0}^{\infty}(k+1)^{-2} e^{i k \beta} .
$$

The pointwise product $F g \in L^{2}([-\pi, \pi))$, and its $m$ th Fourier coefficient is 


$$
(\widehat{F g})_{m}=\frac{1}{2 \pi} \int_{-\pi}^{\pi} F\left(e^{i \beta}\right) g\left(e^{i \beta}\right) e^{-i m \beta} d \beta=\sum_{k=0}^{\infty}(k+1)^{-2} \hat{F}_{m-k}
$$

set

$$
D=\left(\sum_{m=0}^{\infty}\left|(\widehat{F g})_{m}\right|^{2}\right)^{1 / 2}
$$

For any $X \in \mathscr{L}^{+}[\mathscr{S}(\mathbf{R})]^{\prime}$ and $m, n>0$, we have

$$
\begin{aligned}
\left|\left\langle h_{m}, \Delta\left(\mathfrak{f}_{\text {ang }}\right) X h_{n}\right\rangle\right| & \leq \sum_{k=0}^{\infty}\left|\left\langle h_{m}, \Delta\left(\mathfrak{f}_{\text {ang }}\right) h_{k}\right\rangle\right|\left|\left\langle h_{k}, X h_{n}\right\rangle\right| \\
& \leq K \sum_{k=0}^{\infty}(n+1)^{-2}(k+1)^{-2} \hat{F}_{m-k}\left\|(N+1)^{2} X(N+1)^{2}\right\| \\
& =K(n+1)^{-2}(\widehat{F g})_{m}\left\|(N+1)^{2} X(N+1)^{2}\right\| .
\end{aligned}
$$

We deduce from this that the norm

$$
\left\|\Delta\left(\mathfrak{f}_{\text {ang }}\right) X h_{n}\right\| \leq K D(n+1)^{-2}\left\|(N+1)^{2} X(N+1)^{2}\right\|
$$

for all $n \geq 0$.

If we consider the action of $\Delta\left(\mathfrak{f}_{\text {ang }}\right) X$ on finite linear combinations of Hermite functions and then go to the limit, we find that

$$
\left\|\Delta\left(\mathfrak{f}_{\text {ang }}\right) X f\right\| \leq \frac{\pi^{2}}{\sqrt{90}} K D\left\|(N+1)^{2} X(N+1)^{2}\right\|\|f\|, \quad f \in \mathscr{S}(\mathbf{R}) ;
$$

hence $\Delta\left(\mathfrak{f}_{\text {ang }}\right) X$ is bounded. Moreover, its closure is trace class, with

$$
\left\|\overline{\Delta\left(\mathfrak{f}_{\text {ang }}\right) X}\right\|_{\text {tr }} \leq \sum_{n=0}^{\infty}\left\|\Delta\left(\mathfrak{f}_{\text {ang }}\right) X h_{n}\right\| \leq \frac{\pi^{2}}{6} K D\left\|(N+1)^{2} X(N+1)^{2}\right\| .
$$

Also, it is easy to show that the range of $\Delta\left(\mathfrak{f}_{\text {ang }}\right)$ is contained in the domain of $X^{*}$, that $X^{*} \Delta\left(\mathfrak{f}_{\text {ang }}\right)$ is a closable map with trace class closure, and that

$$
\overline{X^{*} \Delta\left(\mathfrak{f}_{\text {ang }}\right)}=\left(\overline{\Delta\left(\mathfrak{f}_{\text {ang }}\right) X}\right)^{*}
$$

The proof of this is simpler and we omit it.

Now we must show that the two traces are equal. In the chain of equalities below, we can use some of the above inequalities to legitimize the interchange of summation order used below.

Then 


$$
\begin{aligned}
\operatorname{tr}\left[\overline{\left(X^{+}\right)^{*} \Delta\left(\mathfrak{f}_{\text {ang }}\right)}\right] & =\overline{\sum_{n \geq 0}\left\langle h_{n}, \Delta\left(\tilde{\mathfrak{f}}_{\text {ang }}\right) X^{+} h_{n}\right\rangle} \\
& =\sum_{n \geq 0}\left[\sum_{m \geq 0}\left\langle X^{+} h_{n}, h_{m}\right\rangle\left\langle h_{m}, \Delta\left(\mathfrak{f}_{\text {ang }}\right) h_{n}\right\rangle\right] \\
& =\sum_{m \geq 0}\left[\sum_{n \geq 0}\left\langle h_{n}, X h_{m}\right\rangle\left\langle\Delta\left(\tilde{f}_{\text {ang }}\right) h_{m}, h_{n}\right\rangle\right] \\
& =\sum_{m \geq 0}\left\langle\Delta\left(\mathfrak{f}_{\text {ang }}\right) h_{m}, X h_{m}\right\rangle \\
& =\sum_{m \geq 0}\left\langle h_{m}, \overline{\Delta\left(\mathfrak{f}_{\text {ang }}\right) X} h_{m}\right\rangle \\
& =\operatorname{tr}\left[\overline{\Delta\left(\mathfrak{f}_{\text {ang }}\right) X}\right] .
\end{aligned}
$$

We note without proof the following extension of this result, shown in a similar fashion:

Corollary 4.4. With the same notation as in the previous proposition, let

$$
P_{2}\left(\mathfrak{f}_{\text {ang }} ; X\right)=\overline{\overline{\Delta\left(\mathfrak{f}_{\text {ang }}\right) X} \Delta\left(\mathfrak{f}_{\text {ang }}\right)} .
$$

Then $P_{2}\left(\mathfrak{f}_{\text {ang }} ; X\right)$ is a linear map from $\mathscr{L}^{+}[\mathscr{S}(\mathbf{R})]^{\prime}$ to the trace class operators on $L^{2}(\mathbb{R})$, with

$$
P_{2}\left(\mathfrak{f}_{\text {ang }} ; X\right)=\left[\Delta\left(\mathfrak{f}_{\text {ang }}\right)^{*} X^{*} \Delta\left(\mathfrak{f}_{\text {ang }}\right)^{*}\right]^{*} .
$$

We then define

$$
\operatorname{Exp}\left[\Delta\left(\tilde{\mathfrak{f}}_{\text {ang }}\right) ; \Delta\left(\mathfrak{f}_{\text {ang }}\right) ; X\right]=\operatorname{tr}\left[P_{2}\left(\mathfrak{f}_{\text {ang }}: X\right)\right]
$$

to be the expectation of the square of $\Delta\left(\mathfrak{f}_{\mathrm{ang}}\right)$ in the state $X$.

In special cases where $f$ leads to operators in $\mathscr{L}^{+}[\mathscr{S}(\mathbf{R})]$, these expectations reduce to the usual expressions.

By using these last two results, presumably one could compute the standard deviations for the number and phase operators, and so test for an uncertainty relation, but we have not done so. In this regard, Carruthers and Nieto point out that if $\left(^{\prime}\right)$ is the natural frequency of an oscillator, $\Delta(\varphi) / \omega$ is a "time operator" for the oscillator, and $\hbar \omega N$ is its energy operator. Then the above calculation would lead to some sort of energy/time uncertainty relation. As this is a rather contentious issue, we prefer not to pursue the matter here, save to note that such an enquiry is possible if one is so minded. 


\section{§5. Boundedness of Angle Operators}

In Proposition 3.6 we gave a necessary condition on $\mathfrak{f}$ in order that $\Delta\left(\mathfrak{f}_{\text {ang }}\right)$ be bounded, based on considerations of its matrix representation. This is not the strongest result possible, and the phase operator, for one, does not satisfy it. In this section we shall improve this result by considering the kernel representation of $\Delta\left(\mathfrak{f}_{\text {ang }}\right)$. The new result will cover $\Delta(\varphi)$, but for this important operator we will proceed directly and get a sharper bound yet.

We begin by considering a singular kernel which appears in subsequent calculations.

Proposition 5.1. The equation

$$
[K u](x)=\int_{x}^{\infty} \frac{u(y)}{y+x} d y, \quad u \in L^{2}(0, \infty), x>0
$$

defines a bounded linear operator $K \in \mathscr{L}\left[L^{2}(0, \infty)\right]$ with norm

$$
\|K\| \leq \frac{1}{2} \pi
$$

Proof. Consider the measurable positive function $\kappa:(0, \infty) \times(0, \infty) \rightarrow$ $[0, \infty)$ given by

$$
\kappa(x, y)= \begin{cases}(x+y)^{-1} & \text { if } 0<x<y \\ 0 & \text { if } 0<y \leq x\end{cases}
$$

Since

$$
\int_{0}^{\infty} x^{-1 / 2} \kappa(x, y) d x=\frac{1}{2} \pi y^{-1 / 2}, \quad y>0,
$$

and

$$
\int_{\mathbf{R}} y^{-1 / 2} \kappa(x, y) d y=\frac{1}{2} \pi x^{-1 / 2}, \quad x>0,
$$

by the Schur test [42], we deduce that the operator $K$ is well defined and bounded. The bound 5.1.b is trivial to verify.

Corollary 5.2. Let $P_{ \pm} \in \mathscr{L}\left[L^{2}(\mathbf{R}), L^{2}(0, \infty)\right]$ be given by

$$
\begin{array}{ll}
{\left[P_{+} g\right](x)=g(x),} & x>0, g \in L^{2}(\mathbb{R}), \\
{\left[P_{-} g\right](x)=g(-x),} & x>0, g \in L^{2}(\mathbb{R}) .
\end{array}
$$

Define the operator $\hat{K}$ on $L^{2}(\mathbb{R})$ by setting 


$$
[\hat{K} g](x)= \begin{cases}{\left[K P_{-} g\right](x)} & \text { if } x>0, \\ 0 & \text { if } x=0, \quad g \in L^{2}(\mathbf{R}) . \\ {\left[K P_{+} g\right](-x)} & \text { if } x<0,\end{cases}
$$

Then $\hat{K}$ is bounded, with

$$
\|\hat{K}\| \leq \frac{1}{2} \pi
$$

Proof. The result follows from the observation that

$$
\begin{aligned}
\|\hat{K} g\|^{2} & =\left\|K P_{+} g\right\|^{2}+\left\|K P_{-} g\right\|^{2} \\
& \leq \frac{\pi^{2}}{4}\left\|P_{+} g\right\|^{2}+\frac{\pi^{2}}{4}\left\|P_{-} g\right\|^{2} \\
& =\frac{\pi^{2}}{4}\|g\|^{2} .
\end{aligned}
$$

The idea underlying our analysis is to write $\Delta\left(\mathfrak{f}_{\text {ang }}\right)$ as an integral over operators which are the quantizations of characteristic functions of (or sign functions associated with) various wedge-shaped region of phase space. This requires us to consider the problem of quantization of such regions, and the effect of certain transformations of phase space which define elements of the metaplectic representation of the symplectic group $S p(1)$. In a sense, then, we are employing what might be termed the method of integral geometry on phase space. We believe that this method is amenable to considerable generalization, and of interest in its own right.

To begin with, we consider the quantization of the phase space distribution sign $\otimes$ sign, which places a \pm 1 in each of the four quadrants: a rectangular checkerboard distribution.

Proposition 5.3. The operator $\Delta(\operatorname{sign} \otimes \operatorname{sign})$ is bounded, with

$$
\|\Delta(\operatorname{sign} \otimes \operatorname{sign})\| \leq 2 .
$$

Proof. Given $f, g \in \mathscr{S}(\mathbf{R})$, it will be convenient to define $F \in \mathscr{S}\left(\mathbf{R}^{2}\right)$ and $G \in \mathscr{S}(\mathbf{R})$ by setting

$$
\begin{gathered}
F(x, y)=\overline{\left(y+\frac{1}{2} x\right)} g\left(y-\frac{1}{2} x\right), \\
G(x)=\int_{\mathbf{R}} \operatorname{sign}(y) F(x, y) d y .
\end{gathered}
$$

Note that 


$$
\begin{aligned}
(\Delta(\operatorname{sign} \otimes \operatorname{sign}) g)(\bar{f}) & =\int_{\Pi} \operatorname{sign}(p) \operatorname{sign}(q) \mathscr{G}(\bar{f} \otimes g)(p, q) d p d q \\
& =\frac{1}{\sqrt{2 \pi}} \int_{\Pi} \operatorname{sign}(p) \operatorname{sign}(q)\left[\mathscr{F}_{1}^{-1} F\right](p, q) d p d q \\
& =\frac{1}{\sqrt{2 \pi}} \int_{\mathbb{R}} \operatorname{sign}(p)\left[\mathscr{F}^{-1} G\right](p) d p \\
& =\frac{i}{\pi} \lim _{L \rightarrow \infty}\left\langle\mathscr{F}-1 g_{I(L)}, \mathscr{F}^{-1} G\right\rangle \\
& =\frac{i}{\pi} \lim _{L \rightarrow \infty}\left\langle g_{I(L)}, G\right\rangle .
\end{aligned}
$$

Now for $L>1$, we can apply Fubini's theorem and rearrange matters to see that

$$
\begin{aligned}
\lim _{L \rightarrow \infty}\langle & \left.g_{I(L)}, G\right\rangle \\
= & \int_{\mathbb{R}^{2}} g_{I(L)}(x) \operatorname{sign}(y) F(x, y) d x d y \\
= & \int_{\mathbb{R}^{2}} g_{I(L)}(x-y) \operatorname{sign}(x+y) \overline{f(x)} g(y) d x d y \\
= & \int_{0}^{\infty} \overline{f(x)}\left(\int_{\mathbb{R}} g_{I(L)}(x-y) g(y) d y-2 \int_{-\infty}^{-x} g_{I(L)}(x-y) g(y) d y\right) d x \\
& +\int_{-\infty}^{0} \overline{f(x)}\left(-\int_{\mathbb{R}} g_{I(L)}(x-y) g(y) d y+2 \int_{-x}^{x} g_{I(L)}(x-y) g(y) d y\right) d x \\
= & \left\langle\operatorname{sign}(Q) f, g_{I(L)} * g\right\rangle-2 \int_{0}^{\infty} \overline{f(x)}\left(\int_{x}^{\infty} g_{I(L)}(x+y)[P-g](y) d y\right) d x \\
& -2 \int_{-\infty}^{0} \overline{f(x)}\left(\int_{-x}^{\infty} g_{I(L)}(-x+y)\left[P_{+} g\right](y) d y\right) d x .
\end{aligned}
$$

The asterisk indicates convolution, as usual.

To proceed further we must apply the dominated convergence theorem several times, and it will save space to refer to it as the $D C T$. Then since for $L>1$ and $0<x<y$, 


$$
\left|g_{I(L)}(x+y)\left[P_{-} g\right](y)\right| \leq(x+y)^{-1}\left|P_{-} g(y)\right|
$$

and

$$
\lim _{L \rightarrow \infty} g_{I(L)}(x+y)\left[P_{-} g\right](y)=(x+y)^{-1} P_{-} g(y),
$$

the $D C T$ implies that

$$
\left|\int_{x}^{\infty} g_{I(L)}(x+y)\left[P_{-} g\right](y) d y\right| \leq\left[K\left|P_{-} g\right|\right](x), \quad L>1, x>0,
$$

and

$$
\lim _{L \rightarrow \infty} \int_{x}^{\infty} g_{I(L)}(x+y)\left[P_{-} g\right](y) d y=\left[K P_{-} g\right](x)=[\hat{K} g](x), \quad x>0 .
$$

Another application of the $D C T$ now yields

$$
\lim _{L \rightarrow \infty} \int_{0}^{\infty} \overline{f(x)}\left(\int_{x}^{\infty} g_{I(L)}(x+y)\left[P_{-} g\right](y) d y\right) d x=\int_{0}^{\infty} \overline{f(x)}\left[K P_{-} g\right](x) d x
$$

Similar analysis of the other integral leads to

$$
\begin{aligned}
(\Delta(\operatorname{sign} \otimes \operatorname{sign}) g)(\bar{f}) & =\frac{i}{\pi} \lim _{L \rightarrow \infty}\left\langle\operatorname{sign}(Q) f, g_{I(L)} * g\right\rangle-\frac{2 i}{\pi}\langle f, \hat{K} g\rangle \\
& =i\langle\operatorname{sign}(Q) f, \mathscr{H} g\rangle-\frac{2 i}{\pi}\langle f, \hat{K} g\rangle .
\end{aligned}
$$

Here $\mathscr{H}$ is the Hilbert transform

$$
\mathscr{H}(f)(x)=\lim _{L \rightarrow \infty} \frac{1}{\pi} g_{I(L)} * f(x)=\frac{1}{\pi} \operatorname{Pv} \int_{\mathbf{R}} \frac{f(y)}{x-y} d y, \quad \text { a.e., } f \in L^{2}(\mathbf{R}) .
$$

Thus

$$
\Delta(\operatorname{sign} \otimes \operatorname{sign})=i \operatorname{sign}(Q) \circ \mathscr{H}-\frac{2 i}{\pi} \hat{K}
$$

is bounded, and $\|\Delta(\operatorname{sign} \otimes \operatorname{sign})\| \leq 2$.

We now use the symplectic group to distort $\operatorname{sign} \otimes$ sign to an oblique checkerboard distribution: 


$$
D_{\alpha}(r \cos \beta, r \sin \beta)= \begin{cases}1 & \text { if } r>0,0 \leq \beta \leq \alpha \\ 1 & \text { if } r>0,-\pi \leq \beta \leq-\pi+\alpha \\ -1 & \text { if } r>0, \alpha<\beta<\pi, \\ -1 & \text { if } r>0,-\pi+\alpha<\beta<0, \\ 0 & \text { if } r=0 .\end{cases}
$$

Proposition 5.4. For any $0 \leq \alpha \leq \pi, D_{\alpha} \in L^{\infty}(\Pi)$,

$$
D_{0}=-1, \quad D_{\pi / 2}=\text { sign } \otimes \operatorname{sign}, \quad D_{\pi}=1,
$$

so that

$$
\Delta\left(D_{0}\right)=-I, \quad \Delta\left(D_{\pi}\right)=I,
$$

and

$$
\left\|\Delta\left(D_{0}\right)\right\|=\left\|\Delta\left(D_{\pi}\right)\right\|=1 .
$$

More generally, $\Delta\left(D_{\alpha}\right)$ is bounded, with

$$
\left\|\Delta\left(D_{\alpha}\right)\right\| \leq 2 .
$$

Proof. The statements concerning the angles $0, \pi / 2$ and $\pi$ are obvious. For general $\alpha$ we begin by introducing the unitary operator of the metaplectic representation given by

$$
\left[U_{\xi} g\right](x)=e^{-\frac{1}{2} i x^{2}} g(x), \quad g \in L^{2}(\mathbf{R}), \quad \xi \in \mathbf{R},
$$

and note that it maps $\mathscr{S}(\mathbf{R})$ to itself for each $\xi$.

A direct calculation gives

$$
\left[\mathscr{G}\left(\overline{U_{\xi} f} \otimes U_{\xi} g\right)\right](p, q)=[\mathscr{G}(\bar{f} \otimes g)](p+\xi q, q)
$$

for $(p, q) \in \Pi$ and $f, g \in \mathscr{S}(\mathbf{R})$. This gives us a basic transformation scheme from the rectangular to the oblique checkerboard distribution:

$$
D_{\pi / 2-\arctan \xi}(q, p)=\operatorname{sign}(p-\xi q) \quad \operatorname{sign}(q), \quad \text { a.e. }
$$

Then

$$
\begin{aligned}
\left\langle U_{\xi} f, \Delta(\operatorname{sign} \otimes \operatorname{sign}) U_{\xi} g\right\rangle & =\int_{\Pi} \operatorname{sign}(p) \operatorname{sign}(q)[\mathscr{G}(\bar{f} \otimes g)](p+\xi q, q) d p d q \\
& =\int_{\Pi} D_{\pi / 2-\arctan \xi}(q, p)[\mathscr{G}(\bar{f} \otimes g)](p, q) d p d q \\
& =\left[\Delta\left(D_{\pi / 2-\arctan \xi}\right) g\right](\bar{f}) .
\end{aligned}
$$


Thus we see that $\Delta\left(D_{\alpha}\right)$ is a unitary transform of $\Delta(\operatorname{sign} \otimes$ sign $)$, with

$$
\Delta\left(D_{\alpha}\right)=U_{\cot \alpha}^{*} \circ \Delta(\operatorname{sign} \otimes \operatorname{sign}) \circ U_{\cot \alpha} .
$$

Hence we have the bound

$$
\left\|\Delta\left(D_{\alpha}\right)\right\| \leq 2
$$

for $0<\alpha<\pi$.

We can write $\Delta\left(\mathfrak{f}_{\text {ang }}\right)$, and in particular $\Delta(\varphi)$ as an integral over $\Delta\left(D_{\alpha}\right)$ plus the Fourier transform of the Hilbert transform. To begin with, it is convenient to know that the basic radial integral is a continuous function of angle.

Lemma 5.5. If $f, g \in \mathscr{S}(\mathbf{R})$, define $Q_{f . g}:[-\pi, \pi] \rightarrow \mathbf{C}$ by

$$
Q_{f, g}(\beta)=\int_{0}^{\infty}[\mathscr{G}(\bar{f} \otimes g)](r \cos \beta, r \sin \beta) r d r, \quad-\pi \leq \beta \leq \pi .
$$

Then $Q_{f, g}$ is continuous.

Proof. Define $X$ by

$$
X=\sup _{(p, q) \in \Pi}\left|\left(1+p^{2}+q^{2}\right)^{2}[\mathscr{G}(\bar{f} \otimes g)](p, q)\right| .
$$

Then

$$
|[\mathscr{G}(\bar{f} \otimes g)](r \cos \beta, r \sin \beta)| \leq X\left(1+r^{2}\right)^{-2}
$$

for $r>0$ and $-\pi \leq \beta<\pi$. Using the fact that $\mathscr{G}(\bar{f} \otimes g)$ is continuous, and applying the $D C T$, it is clear that $Q_{f . g} \in \mathscr{C}[-\pi, \pi]$.

This result enables us to prove that $\Delta\left(D_{\alpha}\right)$ is weakly continuous.

Lemma 5.6. For all $f, g \in \mathscr{S}(\mathbf{R}),\left\langle f, \Delta\left(D_{\alpha}\right) g\right\rangle$ belongs to $\mathscr{C}[0, \pi]$ as a function of $\alpha$, and

$$
\left\|\left\langle f, \Delta\left(D_{\alpha}\right) g\right\rangle\right\| \leq 2\|f\|\|g\| \text {. }
$$

Proof. This follows from writing, in an obvious notation,

$$
\left\langle f, \Delta\left(D_{\alpha}\right) g\right\rangle=\left(\int_{-\pi}^{-\pi+\alpha}-\int_{-\pi+\alpha}^{0}+\int_{0}^{\alpha}-\int_{\alpha}^{\pi}\right) Q_{f . g}(\beta) d \beta .
$$

For the phase operator we have the following representation and bound.

Proposition 5.7. The phase operator may be expressed in terms of the operator $\Delta\left(D_{\alpha}\right)$ by writing 


$$
[\Delta(\varphi) g](\bar{f})=\frac{1}{2} \pi \int_{\mathbf{R}} \operatorname{sign}(q) \overline{f(q)} g(q) d q-\frac{1}{2} \int_{0}^{\pi}\left\langle f, \Delta\left(D_{\alpha}\right) g\right\rangle d \alpha
$$

from which it follows that it is bounded, with

$$
\|\Delta(\varphi)\| \leq \frac{3}{2} \pi
$$

Proof. We integrate (5.18) over $\alpha$ and rearrange, and find that

$$
\begin{aligned}
\int_{0}^{\pi}\left\langle f, \Delta\left(D_{\alpha}\right) g\right\rangle d \alpha \\
\quad=\int_{0}^{\pi}(\pi-2 \beta) Q_{f, g}(\beta) d \beta+\int_{-\pi}^{0}(-\pi-2 \beta) Q_{f, g}(\beta) d \beta \\
\quad=\int_{-\pi}^{\pi}[\pi \operatorname{sign}(\beta)-2 \beta] Q_{f, g}(\beta) d \beta \\
\quad=\pi \int_{\Pi} \operatorname{sign}(q)[\mathscr{G}(\bar{f} \otimes g)](p, q) d p d q-2 \int_{\Pi} \varphi(p, q)[\mathscr{G}(\bar{f} \otimes g)](p, q) d p d q \\
=\pi \int_{\mathbf{R}} \operatorname{sign}(q) \overline{f(q)} g(q) d q-2[\Delta(\varphi) g](\bar{f}),
\end{aligned}
$$

from which the result is immediate.

We shall now use a similar approach in the general case. Naturally the details are a bit more involved. We begin by using the results for the sign function to study the characteristic function of the second and third quadrants.

Lemma 5.8. The quadrant functions can be expressed in terms of the sign function as

$$
\begin{gathered}
\chi_{(-\infty .0)} \otimes \chi_{(0, x)}=\frac{1}{4}[1 \otimes 1-\operatorname{sign} \otimes 1+1 \otimes \operatorname{sign}-\operatorname{sign} \otimes \operatorname{sign}], \\
\chi_{(-x .0)} \otimes \chi_{(-\infty .0)}=\frac{1}{4}[1 \otimes 1-\operatorname{sign} \otimes 1-1 \otimes \operatorname{sign}+\operatorname{sign} \otimes \operatorname{sign}],
\end{gathered}
$$

so that

$$
\Delta\left(\chi_{(-x, 0)} \otimes \chi_{(0, x)}\right)=\frac{1}{4}[I-\operatorname{sign}(P)+\operatorname{sign}(Q)-\Delta(\operatorname{sign} \otimes \operatorname{sign})],
$$




$$
\Delta\left(\chi_{(-\infty, 0)} \otimes \chi_{(-\infty, 0)}\right)=\frac{1}{4}[I-\operatorname{sign}(P)-\operatorname{sign}(Q)+\Delta(\operatorname{sign} \otimes \operatorname{sign})] .
$$

Hence $\Delta\left(\chi_{(-\infty, 0)} \otimes \chi_{(0, \infty)}\right)$ and $\Delta\left(\chi_{(-\infty, 0)} \otimes \chi_{(-\infty .0)}\right)$ are bounded, with

$$
\left\|\Delta\left(\chi_{(-\infty, 0)} \otimes \chi_{(0, x)}\right)\right\| \leq 5 / 4, \quad\left\|\Delta\left(\chi_{(-\infty, 0)} \otimes \chi_{(-\infty .0)}\right)\right\| \leq 5 / 4 .
$$

We shall need the wedge distributions as well: for $0 \leq \alpha \leq \pi$ we define

$$
E_{\alpha}(r \cos \beta, r \sin \beta)= \begin{cases}1 & \text { if } r>0, \alpha \leq \beta \leq \pi, \\ 0 & \text { otherwise }\end{cases}
$$

and

$$
F_{\alpha}(p, q)=E_{\alpha}(p,-q) .
$$

We summarize the properties of these distributions and their quantizations in the next proposition, which is proved by the same methods we used previously.

Proposition 5.9. For the angles $0, \pi / 2$ and $\pi$ we have

$$
E_{0}=1 \otimes \chi_{(0, \infty)}, \quad E_{\pi / 2}=\chi_{(-\infty, 0)} \otimes \chi_{(0, x)}, \quad E_{\pi}=0,
$$

and

$$
F_{0}=1 \otimes \chi_{(-\infty, 0)}, \quad F_{\pi / 2}=\chi_{(-\infty, 0)} \otimes \chi_{(-\infty, 0)}, \quad F_{\pi}=0,
$$

almost everywhere. For their quantizations we have

$$
\Delta\left(E_{0}\right)=\chi_{(0, \infty)}(Q), \quad \Delta\left(E_{\pi / 2}\right)=\Delta\left(\chi_{(-\infty .0)} \otimes \chi_{(0, \infty)}\right), \quad \Delta\left(E_{\pi}\right)=0,
$$

and

$$
\Delta\left(F_{0}\right)=\chi_{(-x, 0)}(Q), \quad \Delta\left(F_{\pi / 2}\right)=\Delta\left(\chi_{(-\infty, 0)} \otimes \chi_{(-x, 0)}\right), \quad \Delta\left(F_{\pi}\right)=0 .
$$

Hence all these operators are bounded, with

$$
\left\|\Delta\left(E_{0}\right)\right\|=\left\|\Delta\left(F_{0}\right)\right\|=1, \quad\left\|\Delta\left(E_{\pi / 2}\right)\right\|,\left\|\Delta\left(F_{\pi / 2}\right)\right\| \leq 5 / 4, \quad\left\|\Delta\left(E_{\pi}\right)\right\|=\left\|\Delta\left(F_{\pi}\right)\right\|=0 .
$$

Applying the metaplectic unitary transformation used above yields

$$
\begin{aligned}
& U_{\xi}^{*} \Delta\left(E_{\pi / 2}\right) U_{\xi}=\Delta\left(E_{\pi / 2-\arctan \xi}\right) \\
& U_{\xi}^{*} \Delta\left(F_{\pi / 2}\right) U_{\xi}=\Delta\left(F_{\pi / 2+\arctan \xi}\right)
\end{aligned}
$$

for $\xi \in \mathbf{R}$. Thus $E_{\alpha}$ and $F_{\alpha}$ are bounded, with

$$
\left\|E_{\alpha}\right\|,\left\|F_{\alpha}\right\| \leq 5 / 4
$$

for $0<\alpha<\pi$.

We are now ready to present our general result on boundedness. 
Proposition 5.10. If $f \in \mathscr{C}^{1}[-\pi, \pi]$, then

$\left[\Delta\left(\mathfrak{f}_{\text {ang }}\right) g\right](\bar{f})=\tilde{f}(0)\langle f, g\rangle+\int_{0}^{+\pi} \tilde{f}^{\prime}(\alpha)\left\langle f, \Delta\left(E_{\alpha}\right) g\right\rangle d \alpha-\int_{0}^{+\pi} f^{\prime}(-\alpha)\left\langle f, \Delta\left(F_{\alpha}\right) g\right\rangle d \alpha$.

Then $\Delta\left(\mathfrak{f}_{\text {ang }}\right)$ is bounded, with

$$
\left\|\Delta\left(\mathfrak{f}_{\text {ang }}\right)\right\| \leq|\mathfrak{f}(0)|+\frac{5}{4} \int_{-\pi}^{+\pi}\left|\mathfrak{f}^{\prime}(\alpha)\right| d \alpha .
$$

Proof. Our strategy will be recognized as following the proof for the phase operator. Under the stated conditions, for $f, g \in \mathscr{S}(\mathbf{R})$ the functions

$$
\alpha \longrightarrow\left\langle f, \Delta\left(E_{\alpha}\right) g\right\rangle \text { and } \alpha \longrightarrow\left\langle f, \Delta\left(F_{\alpha}\right) g\right\rangle
$$

are continuous for $0 \leq \alpha \leq \pi$; and for these angles,

$$
\left\langle f, \Delta\left(E_{\alpha}\right) g\right\rangle=\int_{\alpha}^{\pi} Q_{f, g}(\beta) d \beta \quad \text { and }\left\langle f, \Delta\left(F_{\alpha}\right) g\right\rangle=\int_{-\pi}^{-\alpha} Q_{f, g}(\beta) d \beta .
$$

Moreover, the bounds

$$
\left|\left\langle f, \Delta\left(E_{\alpha}\right) g\right\rangle\right|,\left|\left\langle f, \Delta\left(F_{\alpha}\right) g\right\rangle\right| \leq \frac{5}{4}\|f\|\|g\|
$$

hold.

Fubini's theorem can now be used to interchange order of integration in

$$
\begin{aligned}
\int_{0}^{\pi} \mathfrak{f}^{\prime}(\alpha)\left\langle f, \Delta\left(E_{\alpha}\right) g\right\rangle d \alpha & =\int_{0}^{\pi} \mathfrak{f}^{\prime}(\alpha)\left(\int_{\alpha}^{\pi} Q_{f . g}(\beta) d \beta\right) d \alpha \\
& =\int_{0}^{\pi}\left(\int_{0}^{\beta} \mathfrak{f}^{\prime}(\alpha) d \alpha\right) Q_{f . g}(\beta) d \beta \\
& =\int_{0}^{\pi}[\mathfrak{f}(\beta)-\mathfrak{f}(0)] Q_{f, g}(\beta) d \beta .
\end{aligned}
$$

A similar calculation works for $\Delta\left(F_{\alpha}\right)$, yielding

$$
\int_{0}^{\pi} \mathfrak{f}^{\prime}(-\alpha)\left\langle f, \Delta\left(F_{\alpha}\right) g\right\rangle d \alpha=-\int_{-\pi}^{0}[\mathfrak{f}(\beta)-\mathfrak{f}(0)] Q_{f, g}(\beta) d \beta .
$$

Subtracting these two results gives the representation for $\Delta\left(\tilde{\mathfrak{T}}_{\text {ang }}\right)$. As we have bounds for all the terms in the representation, we find that 


$$
\begin{aligned}
\left|\left[\Delta\left(\mathfrak{f}_{\text {ang }}\right) g\right](\bar{f})\right| \leq & |\mathfrak{f}(0)|\|f\|\|g\|+\frac{5}{4}\left(\int_{0}^{\pi}\left|\tilde{f}^{\prime}(\alpha)\right| d \alpha\right)\|f\|\|g\| \\
& +\frac{5}{4}\left(\int_{0}^{\pi}\left|\mathfrak{f}^{\prime}(-\alpha)\right| d \alpha\right)\|f\|\|g\| \\
= & \left(|\tilde{\mathfrak{f}}(0)|+\frac{5}{4} \int_{-\pi}^{+\pi}\left|\mathfrak{f}^{\prime}(\alpha)\right| d \alpha\right)\|f\|\|g\|,
\end{aligned}
$$

from which the bound on $\Delta\left(\mathfrak{f}_{\text {ang }}\right)$ is immediate.

The connection between this result and Proposition 3.5 needs clarification. Let us observe that if $\mathfrak{f} \in \mathscr{C}^{1}[-\pi, \pi]$ and if

$$
\mathfrak{f}(-\pi)=\mathfrak{f}(\pi),
$$

a simple calculation shows that

$$
\hat{\mathfrak{f}}_{n}=\frac{1}{\text { in }} \hat{\mathfrak{f}}_{n}^{\prime}, \quad n \neq 0
$$

As the sequence $\left(\hat{\mathfrak{f}}_{n}^{\prime}\right)_{\mathbf{Z}}$ is in $l^{2}(\mathbf{Z})$ and $\left(n^{-3 / 4}\right)_{\mathbf{N}}$ is in $l^{2}(\mathbf{N})$, then the condition of Proposition 3.5 is satisfied. So for functions in this class, with the cyclical boundary condition, Proposition 3.5 could have told us that $\Delta\left(\tilde{f}_{\text {ang }}\right)$ was bounded.

We know by considering the phase operator that Proposition 3.5 is not necessary, and does not cover all the functions $\mathfrak{f} \in \mathscr{C}^{1}[-\pi, \pi]$. A closer analysis shows that, in fact, Proposition 3.5 covers only those $\mathfrak{f} \in \mathscr{C}^{1}[-\pi, \pi]$ which satisfy the cyclical boundary conditions. In words, it applies to $\mathscr{C}^{1}$ functions on the circle.

\section{§6. Conclusions}

We began by reviewing a number of previous attempts to define a phase operator for systems with a finite number of degrees of freedom. Having given our reasons for considering the situation as unsatisfactory, we proposed to quantize the angle function in phase space. In order to do so we consider Wigner-Weyl quantization in polar coordinates. After recalling some results known for phase space functions of $p$ or $q$ or $r$, in section 3 we set up the machinery for functions of the angle. The basic algebraic scheme is given in Lemma 3.1.

Before considering the phase operator as such, we considered the various operator classes that result from various classes of phase space functions. In 
particular we give necessary and sufficient conditions for functions $f$ of the angle to determine maps $\Delta\left(\mathfrak{f}_{\text {ang }}\right)$ in $\mathscr{L}^{+}\left[\mathscr{S}(\mathbf{R}), L^{2}(\mathbf{R})\right]$ and in $\mathscr{L}^{+}[\mathscr{S}(\mathbf{R})]$, and a sufficient condition for $\Delta\left(\mathfrak{f}_{\text {ang }}\right)$ to be bounded.

The quantization of the phase space exponential functions $e^{ \pm i \varphi}$ determines bounded operators which are shift operators with respect to the Hermite functions. Interestingly, the shift sequence is of a type not considered before. Being shift operators, it is possible to give a complete spectral analysis, and we do so. The spectrum of the operator obtained by quantizing $e^{i \varphi}$ has a nonempty residual component. It is known that the absence of a residual component in the spectrum of a Hamiltonian is sufficient for asymptotic completeness to hold, but the physical significance of its presence here is not clear to us.

Turning to the phase operator itself, we begin by carefully defining the angle function in phase space, taking due care of the branch point at the origin. This singularity simply cannot be overlooked, even in classical mechanics. This point is brought home by our calculating the Poisson bracket between the phase function and the radial function whose quantization is the number operator. We prove that this pair is not canonical. In addition to the canonical term it has further terms which we call secular.

Quantizing the angle function, our method provides us with an infinite matrix representation, taken with respect to the Hermite functions. We are able to show that the operator $\Delta(\varphi) \in \mathscr{L}^{+}\left[\mathscr{S}(\mathbf{R}), L^{2}(\mathbf{R})\right]$, but is not in $\mathscr{L}^{+}[\mathscr{S}(\mathbf{R})]$. We also give both the kernel of this operator and its Fourier transform in Section 4.

In Section 5 we used the kernel representation to prove that if $\tilde{i}$ is of class $\mathscr{C}^{1}[-\pi, \pi]$, then $\Delta\left(\mathfrak{f}_{\text {ang }}\right)$ is bounded. We did this by representing $\Delta\left(\mathfrak{f}_{\text {ang }}\right)$ in terms of the quantizations of the characteristic functions and the sign functions of certain wedge-shaped regions. This method is reminiscent of classical integral geometry. The bound we obtain can be sharpened still further in the case of $\Delta(\varphi)$, and we showed that it was bounded by $3 \pi / 2$.

Since the range of the angle function is $[-\pi, \pi]$, the bound $3 \pi / 2$ is unexpected, and we suspect that it is not the norm.

Some insight into this problem is afforded by a numerical study. In particular, we have considered the matrices $A_{n}$ obtained from $\left\langle h_{i}, \Delta(\varphi) h_{j}\right\rangle$ by setting to zero all matrix elements for $i, j>n$. We may then consider the norm of $A_{n}$ by finding its largest eigenvalue. To this end we have computed $\left\|A_{n}\right\|$ for a number of values of $n$ up to 120 numerically, using Mathematica 2.0 [41] on an Apple Mac IIsi. A few values are given in the table below.

\begin{tabular}{|c|c|r|r|r|r|r|r|r|r|}
\hline$n$ & 0 & 5 & 10 & 20 & 30 & 40 & 58 & 70 & 120 \\
\hline$\left\|A_{n}\right\|$ & 0 & 2.26 & 2.61 & 2.84 & 2.93 & 2.98 & 3.02 & 3.04 & 3.08 \\
\hline
\end{tabular}


A graph will show that the norms seem to approach $\pi$ "asymptotically" very smoothly. The distribution of the other eigenvalues seem to be uniform. As the norm of $\Delta(\varphi)$ is certainly not less than the supremum of the $\left\|A_{n}\right\|$, and in view of the interpretation of $\Delta(\varphi)$, we are led to the make the following conjecture.

Conjecture 6.1. The norm of the phase operator is $\|\Delta(\varphi)\|=\pi$, and its spectrum is purely continuous, with

$$
\sigma(\Delta(\varphi))=\sigma_{\text {cont }}(\Delta(\varphi))=[-\pi, \pi] .
$$

Because the number operator is unbounded, and $\mathscr{S}(\mathbf{R})$ is not stable under the phase operator, we defined the commutator of $\Delta(\varphi)$ and $N$ as a form in Section 4. Just as in the classical case, the commutator consists of both canonical and secular terms. These latter are dependent on $\hbar$, and thus provide a possible source of interesting quantal effects. We also define the relevant expectations, and these, too, could be measured, in principle.

\section{Appendix}

We begin with a more or less standard lemma concerning the Cauchy principal value. To avoid misunderstandings, we note that the integrals are Lebesgue integrals [40].

Lemma A.1. For any $f \in \mathscr{S}(\mathbf{R})$ and $P \in \mathbf{R}$, the limit

$$
I_{P}(f)=\lim _{\varepsilon \rightarrow 0+} \int_{|x| \geq \varepsilon} \frac{f(x) e^{i P x}}{x} d x
$$

exists, and

$$
\begin{gathered}
\lim _{P \rightarrow \infty} I_{P}(f)=\pi i f(0) \\
\lim _{P \rightarrow \infty} I_{-P}(f)=-\pi i f(0) .
\end{gathered}
$$

Proof. We can transfer all integration to the positive real axis by a change of variables :

$$
\begin{aligned}
\int_{|x| \geq \varepsilon} \frac{f(x) e^{i P x}}{x} d x= & \int_{\varepsilon}^{\infty} \frac{f(x)-f(-x)}{x} \cos (P x) d x \\
& +i \int_{\varepsilon}^{\infty} \frac{f(x)+f(-x)}{x} \sin (P x) d x
\end{aligned}
$$

so it follows that $I_{P}(f)$ exists, and we can write 


$$
\begin{aligned}
I_{P}(f)= & \int_{0}^{\infty} \frac{f(x)-f(-x)}{x} \cos (P x) d x+i \int_{0}^{\infty} \frac{f(x)+f(-x)}{x} \sin (P x) d x \\
= & \int_{0}^{\infty} \frac{f(x)-f(-x)}{x} \cos (P x) d x+i \int_{1}^{\infty} \frac{f(x)+f(-x)}{x} \sin (P x) d x \\
& +i \int_{0}^{1} \frac{f(x)+f(-x)-2 f(0)}{x} \sin (P x) d x+2 i f(0) \int_{0}^{1} \frac{\sin (P x)}{x} d x \\
= & \int_{0}^{\infty} \frac{f(x)-f(-x)}{x} \cos (P x) d x+i \int_{1}^{x} \frac{f(x)+f(-x)}{x} \sin (P x) d x \\
& +i \int_{0}^{1} \frac{f(x)+f(-x)-2 f(0)}{x} \sin (P x) d x+2 i f(0) \int_{0}^{P} \frac{\sin x}{x} d x .
\end{aligned}
$$

The limit results are now immediate from the Riemann-Lebesgue lemma.

Lemma A.2. For any $f \in \mathscr{S}(\mathbf{R})$ and $q \neq 0$, we have that

$$
\begin{aligned}
\int_{\mathbf{R}} \varphi(p, q)\left[\mathscr{F}^{-1} f\right](p) d p= & \pi \sqrt{\frac{\pi}{2}} \operatorname{sign}(q) f(0) \\
& -i \sqrt{\frac{\pi}{2}} \operatorname{sign}(q) \int_{0}^{\infty} \frac{f(x)-f(-x)}{x} e^{-x|q|} d x .
\end{aligned}
$$

Proof. Note that for $q \neq 0$,

$$
\left|\int_{-P}^{P} \frac{e^{i p x}}{p^{2}+q^{2}}\right| \leq \frac{\pi}{|q|}, \quad P>0, x \in \mathbf{R},
$$

and that

$$
\lim _{P \rightarrow \infty} \int_{-P}^{P} \frac{e^{i p x}}{p^{2}+q^{2}}=\frac{\pi}{|q|} e^{-x|q|} \quad x \in \mathbf{R}
$$

and also that

$$
\lim _{P \rightarrow \infty} \varphi(P, q)=0 \text { and } \lim _{P \rightarrow \infty} \varphi(-P, q)=\pi \operatorname{sign}(q)
$$

Then

$$
\begin{aligned}
& \int_{-P}^{P} \varphi(p, q)\left[\mathscr{F}^{-1} f\right](p) d p \\
& \quad=(2 \pi)^{-1 / 2} \int_{-P}^{P} \varphi(p, q)\left[\int_{\mathbf{R}} f(x) e^{i p x} d x\right] d p
\end{aligned}
$$




$$
\begin{aligned}
= & (2 \pi)^{-1 / 2} \lim _{\varepsilon \rightarrow 0+} \int_{-P}^{P} \varphi(p, q)\left(\int_{|x| \geq \varepsilon} f(x) e^{i p x} d x\right) d p \\
= & (2 \pi)^{-1 / 2} \lim _{\varepsilon \rightarrow 0+} \int_{|x| \geq \varepsilon} f(x)\left(\int_{-P}^{P} \varphi(p, q) e^{i p x} d p\right) d x \\
= & (2 \pi)^{-1 / 2} \lim _{\varepsilon \rightarrow 0+} \int_{|x| \geq \varepsilon} f(x)\left(\frac{\varphi(P, q) e^{i P x}}{i x}-\frac{\varphi(-P, q) e^{-i P x}}{i x}+\frac{q}{i x} \int_{-P}^{P} \frac{e^{i p x}}{p^{2}+q^{2}}\right) \\
= & -i(2 \pi)^{-1 / 2} \varphi(P, q) I_{P}(f)+i(2 \pi)^{-1 / 2} \varphi(-P, q) I_{-P}(f) \\
& -i(2 \pi)^{-1 / 2} q \lim _{\varepsilon \rightarrow 0+} \int_{\varepsilon}^{\infty} \frac{f(x)-f(-x)}{x}\left(\int_{-P}^{P} \frac{e^{i p x}}{p^{2}+q^{2}} d p\right) d x \\
= & -i(2 \pi)^{-1 / 2} \varphi(P, q) I_{P}(f)+i(2 \pi)^{-1 / 2} \varphi(-P, q) I_{-P}(f) \\
& -i(2 \pi)^{-1 / 2} q \int_{0}^{\infty} \frac{f(x)-f(-x)}{x}\left(\int_{-P}^{P} \frac{e^{i p x}}{p^{2}+q^{2}} d p\right) d x .
\end{aligned}
$$

Applying the dominated convergence theorem and substituting from above gives

$$
\begin{aligned}
\int_{\mathbf{R}} \varphi & (p, q)\left[\mathscr{F}^{-1} f\right](p) d p \\
& =\lim _{P \rightarrow \infty} \int_{-P}^{P} \varphi(p, q)\left[\mathscr{F}^{-1} f\right](p) d p \\
& =\pi \sqrt{\frac{\pi}{2}} \operatorname{sign}(q) f(0)-i \sqrt{\frac{\pi}{2}} \operatorname{sign}(q) \int_{0}^{\infty} \frac{f(x)-f(-x)}{x} e^{-x|q|} d x
\end{aligned}
$$

completing the proof.

For the next step in the derivation of the kernel, define the function $F \in \mathscr{S}(\Pi)$ by setting

Then

$$
F(p, q)=\overline{f\left(q+\frac{1}{2} p\right)} g\left(q-\frac{1}{2} p\right), \quad f, g \in \mathscr{S}(\mathbf{R})
$$

$$
[\mathscr{G}(\bar{f} \otimes g)](p, q)=(2 \pi)^{-1 / 2}\left[\mathscr{F}_{1}^{-1} F\right](p, q),
$$

and the function $p^{-1}[F(p, q)-F(-p, q)]$ is in $L^{1}((0, \infty) \times \mathbf{R})$. Then

$$
\begin{aligned}
\int_{\mathbf{R}} \varphi(p, q)\left[\mathscr{F}^{-1} F\right](p, q) d p= & \pi \sqrt{\frac{\pi}{2}} \operatorname{sign}(q) \overline{f(q)} g(q) \\
& -i \sqrt{\frac{\pi}{2}} \operatorname{sign}(q) \int_{0}^{\infty} \frac{F(x, q)+F(-x, q)}{x} e^{-x|q|} d x
\end{aligned}
$$


for all $q \neq 0$.

Recalling the function $g_{I} \in L^{1} \cap L^{\infty}$ for $L>1$, given in (4.1.b), we see that

$$
\begin{aligned}
\langle f, \Delta(\varphi) g\rangle & (2 \pi)^{-1 / 2} \int_{\Pi} \varphi(p, q)\left[\mathscr{F}^{-1} F\right](p, q) d p d q \\
= & \frac{\pi}{2} \int_{\mathbf{R}} \operatorname{sign}(q) \overline{f(q)} g(q) d q-\frac{i}{2} \int_{\mathbf{R}} \operatorname{sign}(q)\left(\int_{0}^{\infty} \frac{F(x, q)-F(-x, q)}{x} e^{-x|q|} d x\right) d q \\
= & \frac{\pi}{2} \int_{\mathbf{R}} \operatorname{sign}(q) \overline{f(q)} g(q) d q \\
& -\frac{i}{2} \lim _{L \rightarrow \infty} \int_{\mathbf{R}} \operatorname{sign}(q)\left(\int_{1 / L}^{L} \frac{F(x, q)-F(-x, q)}{x} e^{-x|q|} d x\right) d q \\
= & \frac{\pi}{2} \int_{\mathbf{R}} \operatorname{sign}(q) \overline{f(q)} g(q) d q-\frac{i}{2} \lim _{L \rightarrow \infty} \int_{\mathbf{R}^{2}} \operatorname{sign}(q) g_{I(L)}(x) F(x, q) e^{-|x q|} d x d q \\
= & \frac{\pi}{2} \int_{\mathbf{R}} \operatorname{sign}(q) \overline{f(q)} g(q) d q \\
& -\frac{i}{2} \lim _{L \rightarrow \infty} \int_{\Pi} \operatorname{sign}(q) g_{I(L)}(p) \overline{f\left(q+\frac{1}{2} p\right) g\left(q-\frac{1}{2} p\right) e^{-|p q|} d p d q,}
\end{aligned}
$$

which is the expression we require.

In this next part of the appendix we consider the classical Poisson bracket between the number and phase distributions,

$$
\begin{aligned}
& v(f)=\frac{1}{2} \int_{\Pi}\left(p^{2}+q^{2}-1\right) f(p, q) d p d q, \quad f \in \mathscr{S}(\Pi), \\
& \varphi(f)=\int_{\Pi} \varphi(p, q) f(p, q) d p d q, \quad \quad f \in S(\Pi) .
\end{aligned}
$$

It is convenient to work with the distribution $v^{\prime}=v+\frac{1}{2}$. Then integration by parts to clear all derivatives from $g$ gives us

$$
v^{\prime}(\{f, g\})=\int_{\Pi}\left[p \partial_{2} f(p, q)-q \partial_{1} f(p, q)\right] g(p, q) d p d q, \quad f, g \in \mathscr{S}(\Pi) .
$$

This means that $v$ is of such a class that if we extend the Poisson bracket to $\mathscr{S}(\Pi)^{\prime}$ in one variable,

$$
\beta: \mathscr{S}(\Pi)^{\prime} \times \mathscr{S}(\Pi) \longrightarrow \mathscr{S}(\Pi)^{\prime}
$$

by the formula 


$$
[\beta(T, f)](g)=T(\{f, g\}),
$$

then

$$
\beta\left(v^{\prime}, f\right)(p, q)=p \partial_{2} f(p, q)-q \partial_{1} f(p, q)
$$

is not only well defined, but is in $\mathscr{S}(\Pi)$; similarly for $v$ which has the same bracket relations as $v^{\prime}$ does.

This is not true of $\varphi$. Let us evaluate the two terms in this case. For the first term, if $f, g \in \mathscr{S}(\Pi)$ we have

$$
\begin{aligned}
& \int_{\Pi} \varphi(p, q) \partial_{2} f(p, q) \partial_{1} g(p, q) d p d q \\
& \quad=-\lim _{\substack{R \rightarrow \infty \\
\varepsilon \rightarrow 0+}} \int_{\varepsilon<|p|<R} d p \int_{\mathbf{R}}\left[\partial_{1} \varphi(p, q) \partial_{2} f(p, q)+\varphi(p, q) \partial_{1} \partial_{2} f(p, q)\right] g(p, q) d q \\
& \quad=\int_{\Pi}\left[q\left(p^{2}+q^{2}\right)^{-1} \partial_{2} f(p, q)-\varphi(p, q) \partial_{1} \partial_{2} f(p, q)\right] g(p, q) d p d q
\end{aligned}
$$

The second term is

$$
\begin{aligned}
& \int_{\Pi} \varphi(p, q) \partial_{1} f(p, q) \partial_{2} g(p, q) d p d q \\
& =\lim _{\substack{R \rightarrow \infty \\
\varepsilon \rightarrow 0+}} \int_{\mathbf{R}}\left[\left.\varphi(p, q) \partial_{1} f(p, q) g(p, q)\right|_{q=-R} ^{q=+R}-\left.\varphi(p, q) \partial_{1} f(p, q) g(p, q)\right|_{q=-\varepsilon} ^{q=+\varepsilon}\right] d p \\
& \quad-\lim _{\substack{R \rightarrow \infty \\
\varepsilon \rightarrow 0+}} \int_{\mathbf{R}} d p \int_{\varepsilon<|q|<R}\left[\partial_{2} \varphi(p, q) \partial_{1} f(p, q)+\varphi(p, q) \partial_{1} \partial_{2} f(p, q)\right] g(p, q) d q \\
& =-2 \pi \int_{-\infty}^{0} \partial_{1} f(p, 0) g(p, 0) d p \\
& \quad-\int_{\Pi}\left[p\left(p^{2}+q^{2}\right)^{-1} \partial_{1} f(p, q)+\varphi(p, q) \partial_{1} \partial_{2} f(p, q)\right] g(p, q) d p d q .
\end{aligned}
$$

Thus

$$
\begin{aligned}
\beta(\varphi, f)(g)= & -2 \pi \int_{-\infty}^{0} \partial_{1} f(p, 0) g(p, 0) d p \\
& -\int_{\Pi}\left(p^{2}+q^{2}\right)^{-1}\left[p \partial_{1} f(p, q)+q \partial_{2} f(p, q)\right] g(p, q) d p d q .
\end{aligned}
$$

We now extend the Poisson bracket to those pairs of tempered distributions $S, T$ for which 


$$
[\{S, T\}, f]=[S, \beta(T, f)]
$$

is well defined for all $f \in \mathscr{S}(\Pi)$. This so for $\varphi$ and $v^{\prime}$, with

$$
\begin{aligned}
{\left[\left\{\varphi, v^{\prime}\right\}, f\right]=} & -2 \pi \int_{-\infty}^{0} p f(p, 0) d p \\
& -\int_{\Pi}\left(p^{2}+q^{2}\right)^{-1}\left[p \partial_{1} v(p, q)+q \partial_{2} v(p, q)\right] f(p, q) d p d q \\
= & -2 \pi \int_{-\infty}^{0} p f(p, 0) d p-\int_{\Pi} f(p, q) d p d q,
\end{aligned}
$$

which is equivalent to (3.3.a).

\section{References}

[1] Dirac, P. A. M., The Quantum Theory of the Emission and Absorption of Radiation, Proc. Roy. Soc. (London), A114 (1927), 243-265.

[2] Susskind, L. and Glogower, J., Quantum Mechanical Phase and Time Operator, Physics, 1 (1964), 49-61.

[3] Carruthers, P. and Nieto, M. M., Phase and Angle Variables in Quantum Mechanics, Rev. Mod. Phys., 40 (1968), 411-441.

[4] Dubin, D. A. and Hennings, M. A., Quantum mechanics, algebras and distributions, Pitman Research Notes in Mathematics 238, Longman, UK, 1990.

[5] Schmüdgen, K., Unbounded Operator Algebras and Representation Theory, AkademieVerlag, Berlin, 1990.

[6] Garrison, J. C. and Wong, J., Canonically Conjugate Pairs, Uncertainty Relations, and Phase Operators, J. Math. Phy's., 11 (1970), 2242-2249.

[7] Popov, V. N. and Yarunin, V. S., Quantum and quasi-classical states of the photon phase operator, J. Mod. Opt., 39 (1992), 1525-1531.

[8] Galindo, A., Phase and Number, Lett. Math. Phys., 8 (1984), 495-500.

[9] Pegg, D. T., Vaccaro, J. A. and Barnett, S. M., Quantum-Optical Phase and Canonical Conjugation, J. Mod. Opt., 37 (1990), 1703-1710.

[10] Lerner, E. C., Huang H. W. and Walters, G. E., Some Mathematical Properties of Oscillator Phase Operators, J. Math. Phys., 11 (1970), 1679-1684.

[11] Newton, R. G., Quantum Action-Angle Variables for Harmonic Oscillators, Ann. of Phys. (N.Y.), 124 (1980), 327-346.

[12] Ban, M., Phase State and Phase Probability Distribution, Optics Conmunications, 94 (1992), 231-237.

[13] Rocca, F. and Sirugue, M., Phase Operator and Condensed Systems, Comm. Math. Phys., 34 (1973), 111-120.

[14] Dubin, D. A., Bosons in Thermal Contact: A C*-Algebraic Model, Comm. Math. Phys., 32 (1973), 1-17.

[15] Rieckers, F. and Ullrich, M., Extended Gauge Transformations and the Physical Dynamics in a Finite Temperature BCS-Model, Acta Physica Austr., 56 (1985), 131-152.

- Condensed Cooper Pairs and Quasi Particles in a Gauge Invariant Finite Temperature BCS-Model, Acta Physica Austr., 56 (1985), 259-274. 
[16] Dubin, D. A., Solvable Models in Algebraic Statistical Mechanics, Oxford Univ. Press (Clarendon), Oxford, 1974.

[17] Berezin, F. A. and Šbin, M. A., Symbols of Operators and Quantization, Colloquia Math. Soc. J. Bolyai, 5 (1980), 21-52.

[18] Lassner, G. A., Operator Symbols in the Description of Observable-State Systems, Rep. Math. Phy's., 16 (1979), 271-180.

[19] Hansen, F., The Moyal Product and Spectral Theory for a Class of Infinite Dimensional Matrices, Publ. RIMS, Kyoto Univ., 26 (1990), 885-933.

[20] Daubechies, I., On the Distributions Corresponding to Bounded Operators in the Weyl Quantization, Comm. Math. Phys., 75 (1980), 229-238.

[21] Daubechies, I., and Grossmann, A., An Integral Transform Related to Quantization, J. Math. Phy's., 21 (1980), 2080-2090.

[22] Moyal, J. E., Quantum Mechanics as a Statistical Theory, Proc. Cambridge Soc., 45 (1949), 99-124.

[23] deGroot, S. R. and Suttorp, L. G., Foundations of Electrodynamics, North-Holland. Amsterdam, 1972.

[24] Segal, I. E., Transforms for Operators and Symplectic Automorphisms over a Locally Compact Abelian Group, Math. Scand., 13 (1963), 31-43.

[25] Pool, J. C. T., Mathematical Aspects of the Weyl Correspondence, J. Math. Phy's., 7 (1966), 66-76.

[26] Loupias, G., C*-algèbres des systèmes canoniques, II. Ann. Inst. H. Poincaré VI. 1 (1967), 39-58.

[27] Weyl, H., Gruppentheorie und Quantenmechanik, S. Hirzel, Leipzig, 1931.

[28] Wigner, E.P., On the Quantum Correction for Thermodynamic Equilibrium, Phy's. Ret., 40 (1932), 749-759.

[29] Folland, G. B., Harmonic Analysis in Phase Space, Princeton University Press, Princeton, N.J., 1989.

[30] Reed, M. and Simon, B., Methods of Modern Mathematical Physics, I, Academic Press, New York, 1972.

[31] Kato, T., Perturbation Theory for Linear Operators, Springer, New York. 1966.

[32] Smith, T. B., Dubin, D. A. and Hennings, M. A., The Weyl Quantization of Phase Angle, J. Mod. Opt., 39 (1992), 1603-1608.

[33] Klauder, J. R., The Design of Radar Signals Having Both High Range Resolution and High Velocity Resolution, Bell Sy'stem Tech. J., 39 (1960), 809-820.

[34] Itzykson, C., Remarks on Boson Commutation Rules, Comm. Math. Phys., 4 (1967), 92-122.

[35] Miller, W., Lie Theory and Special Functions, Academic Press, New York, 1968.

[36] Vilenkin, N. Ya., Laguerre Polynomials, Whittaker Functions and the Representations of Groups of Bordered Matrices, Math. USSR Sb., 4 (1968), 399-410.

[37] Peetre, J., The Weyl Transform and Laguerre Polynomials, Le Mathematiche (Catania), 27 (1972), 301-323.

[38] Howe, R., Quantum Mechanics and Partial Differential Equations, J. Funct. Anal., 38 (1980), 188-254.

[39] Smith, T. B., Correspondence Limit in the Wigner-Weyl Picture, Phy's. Lett., 95A (1983). 219-222.

[40] Rudin, W., Real and Complex Analysis, Springer, Berlin, 1978.

[41] Wolfram Research, Inc., Mathematica 2.0, Wolfram Research, Inc., Champaign. Ill., 1991.

[42] Halmos, P. R., and Sunder, V. S., Bounded Integral Operators on L2 Spaces, McGraw-Hill, New York, 1974. 
Note added in proof: At the 1993 Wigner Symposium held in Oxford, Royer independently proposed our operator $\Delta(\varphi)$ as a phase operator. Using techniques similar to those of our earlier paper [32], he derived its Hermite matrix elements. He also considered the quantization of the symbol $\varphi$ in quantization schemes other than that of Wigner-Weyl. 